\title{
DISCRETE FOURIER ANALYSIS, CUBATURE AND INTERPOLATION ON A HEXAGON AND A TRIANGLE
}

\author{
HUIYUAN LI, JIACHANG SUN, AND YUAN XU
}

\begin{abstract}
Several problems of trigonometric approximation on a hexagon and a triangle are studied using the discrete Fourier transform and orthogonal polynomials of two variables. A discrete Fourier analysis on the regular hexagon is developed in detail, from which the analysis on the triangle is deduced. The results include cubature formulas and interpolation on these domains. In particular, a trigonometric Lagrange interpolation on a triangle is shown to satisfy an explicit compact formula, which is equivalent to the polynomial interpolation on a planer region bounded by Steiner's hypocycloid. The Lebesgue constant of the interpolation is shown to be in the order of $(\log n)^{2}$. Furthermore, a Gauss cubature is established on the hypocycloid.
\end{abstract}

\section{INTRODUCTION}

Approximation by trigonometric polynomials, or equivalently complex exponentials, is at the root of approximation theory. Many problems central to approximation theory were first studied for trigonometric polynomials on the real line or on the unit circle, as illustrated in the classical treatise of Zygmund [26]. Much of the advance in the theory of trigonometric approximation is due to the periodicity of the function. In the case of one variable, one usually works with $2 \pi$ periodic functions. A straightforward extension to several variables is the tensor product type, where one works with functions that are $2 \pi$ periodic in each of their variables. There are, however, other definitions of periodicity in several variables, most notably the periodicity defined by a lattice, which is a discrete subgroup defined by $A \mathbb{Z}^{d}$, where $A$ is a nonsingular matrix, and the periodic function satisifes $f(x+A k)=f(x)$, for all $k \in \mathbb{Z}^{d}$. With such a periodicity, one works with exponentials or trigonometric functions of the form $\mathrm{e}^{i 2 \pi \alpha \cdot x}$, where $\alpha$ and $x$ are in proper sets of $\mathbb{R}^{d}$, not necessarily the usual trigonometric polynomials.

If $\Omega$ is a bounded open set that tiles $\mathbb{R}^{d}$ with the lattice $L=A \mathbb{Z}^{d}$, meaning that $\Omega+A \mathbb{Z}^{d}=\mathbb{R}^{d}$, then a theorem of Fuglede [6] states that the family of exponentials $\left\{\mathrm{e}^{2 \pi i \alpha \cdot x}: \alpha \in L^{\perp}\right\}$, where $L^{\perp}=A^{-\operatorname{tr}} \mathbb{Z}^{d}$ is the dual lattice of $L$, forms an orthonormal basis in $L^{2}(\Omega)$. In particular, this shows that it is appropriate to use such exponentials for approximation. Naturally one can study Fourier series on $\Omega$, defined by these exponentials, which is more or less the usual multivariate Fourier series with a change of variables from $\mathbb{Z}^{d}$ to $A \mathbb{Z}^{d}$. Lattices appear prominently in

Date: November 5, 2018.

1991 Mathematics Subject Classification. 41A05, 41A10.

Key words and phrases. Discrete Fourier series, trigonometric, Lagrange interpolation, hexagon, triangle, orthogonal polynomials.

The first and the second authors were supported by NSFC Grant 10431050 and 60573023 . The second author was supported by National Basic Research Program Grant 2005CB321702. The third author was supported by NSF Grant DMS-0604056. 
various problems in combinatorics and analysis (see, for example, [2, [5]), the Fourier series associated with them are studied in the context of information science and physics, such as sampling theory [7, 11] and multivariate signal processing [3]. As far as we are aware, they have not been studied much in approximation theory until recently. Part of the reason may lie in the complexity of the set $\Omega$. In the simplest non-tensor product case, it is a regular hexagon region on the plane, not one of those regions that people are used to in classical analysis. It turns out, however, that the results on the hexagon are instrumental for the study of trigonometric functions on the triangle [21, 22]. The Fourier series on the hexagon were studied from scratch in [21], and two families of functions analogues to those of sine and cosine but defined using three directions were identified and studied. In 22 the two generalized trigonometric functions were derived as solutions of the eigenfunctions of the Laplace equation on a triangle, and the Fourier series in terms of them were studied.

The purpose of the present paper is to study discrete Fourier analysis on the hexagon and on the triangle; in particular, we will derive results on cubature and Lagrange interpolation on these domains. We shall follow two approaches. The first one relies on the discrete Fourier transforms associated with the lattices, which leads naturally to an interpolation formula, and the result applies to a general lattice. We then apply the result to the hexagonal tiling. The symmetry group of the hexagon lattice is the reflection group $\mathcal{A}_{2}$. The generalized cosine and sine are invariant and anti-invariant projections, respectively, of the basic exponential function under $\mathcal{A}_{2}$, and they can be studied as functions defined on a fundamental domain, which is one of the equilateral triangles inside the hexagon. This allows us to pass from the hexagon to the triangle. The discrete Fourier transform can be regarded as a cubature formula. In the case of a triangle, the trigonometric functions for which such a cubature holds is a graded algebra due to a connection to polynomials of two variables, which shows that the cubature is a Gaussian type.

The second approach uses orthogonal polynomials in two variables. It turns out that the generalized cosine and sine functions were studied earlier by Koornwinder in [9, who also started with eigenfunctions of the Laplace operator and identified them as orthogonal polynomials on the region bounded by Steiners hypocycloid, which he called Chebyshev polynomials of the first and the second kind, respectively. It is well known that orthogonal polynomials are closely related to cubature formulas and polynomial interpolation (see, for example, 4, 14, 20, 24, 25]). Roughly speaking, a cubature formula of high precision exists if the set of its nodes is an algebraic variety of a polynomial ideal generated by (quasi)orthogonal polynomials, and the size of the variety is equal to the codimension of the ideal. Furthermore, such a cubature formula arises from an interpolation polynomial. The rich structure of the generalized Chebyshev polynomials allow us to study their common zeros and establish the cubature and the polynomial interpolation. One particular result shows that Gaussian cubature formula exists for one integral on the region bounded by the hypocycloid. It should be mentioned that, unlike the case of one variable, Gaussian cubature formulas rarely exist in several variables.

The most concrete result, in our view, is the trigonometric interpolation on the triangle. For a standard triangle $\Delta:=\{(x, y): x \geq 0, y \geq 0, x+y \leq 1\}$, the set of interpolation points are $X_{n}:=\left\{\left(\frac{i}{n}, \frac{j}{n}\right): 0 \leq i \leq j \leq n\right\}$ in $\Delta$. We found a compact formula for the fundamental interpolation functions in terms of the elementary 
trigonometric functions, which ensures that the interpolation can be computed efficiently. Moreover, the uniform operator norm (the Lebesgue constant) of the interpolation is shown to grow in the order of $(\log n)^{2}$ as $n$ goes to infinity. This is in sharp contrast to the algebraic polynomial interpolation on the set $X_{n}$, which also exists and can be given by simple formulas, but has an undesirable convergence behavior (see, for example, [17]). In fact, it is well known that equally spaced points are not good for algebraic polynomial interpolation. For example, a classical result shows that the polynomial interpolation of $f(x)=|x|$ on equally spaced points of $[-1,1]$ diverges at all points other than $-1,0,1$.

The paper is organized as follows. In the next section we state the basic results on lattices and discrete Fourier transform, and establish the connection to the interpolation formula. Section 3 contains results on the regular hexagon, obtained from applying the general result of the previous section. Section 4 studies the generalized sine and cosine functions, and the discrete Fourier analysis on the triangle. In particular, it contains the results on the trigonometric interpolation. The generalized Chebyshev polynomials and their connection to the orthogonal polynomials of two variables are discussed in Section 5, and used to establish the existence of Gaussian cubature, and an alternative way to derive interpolation polynomial.

\section{Continuous and discrete Fourier analysis With lattice}

2.1. Lattice and Fourier series. A lattice in $\mathbb{R}^{d}$ is a discrete subgroup that contains $d$ linearly independent vectors,

$$
L:=\left\{k_{1} a_{1}+k_{2} a_{2}+\cdots+k_{d} a_{d}: \quad k_{i} \in \mathbb{Z}, i=1,2, \ldots, d\right\},
$$

where $a_{1}, \ldots, a_{d}$ are linearly independent column vectors in $\mathbb{R}^{d}$. Let $A$ be the $d \times d$ matrix whose columns are $a_{1}, \ldots, a_{d}$. Then $A$ is called a generator matrix of the lattice $L$. We can write $L$ as $L_{A}$ and a short notation for $L_{A}$ is $A \mathbb{Z}^{d}$; that is,

$$
L_{A}=A \mathbb{Z}^{d}=\left\{A k: k \in \mathbb{Z}^{d}\right\} .
$$

Any $d$-dimensional lattice has a dual lattice $L^{\perp}$ given by

$$
L^{\perp}=\left\{x \in \mathbb{R}^{d}: x \cdot y \in \mathbb{Z} \text { for all } y \in L\right\},
$$

where $x \cdot y$ denote the usual Euclidean inner product of $x$ and $y$. The generator matrix of the dual lattice $L_{A}^{\perp}$ is $A^{-\mathrm{tr}}:=\left(A^{\text {tr }}\right)^{-1}$, where $A^{\text {tr }}$ denote the matrix transpose of $A$ (2]). Throughout this paper whenever we need to treat $x \in \mathbb{R}^{d}$ as a vector, we treat it as a column vector, so that $x \cdot y=x^{\text {tr }} y$.

A bounded set $\Omega$ of $\mathbb{R}^{d}$ is said to tile $\mathbb{R}^{d}$ with the lattice $L$ if

$$
\sum_{\alpha \in L} \chi_{\Omega}(x+\alpha)=1, \quad \text { for almost all } x \in \mathbb{R}^{d},
$$

where $\chi_{\Omega}$ denotes the characteristic function of $\Omega$. We write this as $\Omega+L=\mathbb{R}^{d}$. Tiling and Fourier analysis are closely related; see, for example, [6, 8]. We will need the following result due to Fuglede [6]. Let $\langle\cdot, \cdot\rangle_{\Omega}$ denote the inner product in the space $L^{2}(\Omega)$ defined by

$$
\langle f, g\rangle_{\Omega}=\frac{1}{\operatorname{mes}(\Omega)} \int_{\Omega} f(x) \overline{g(x)} d x .
$$


Theorem 2.1. 6 Let $\Omega$ in $\mathbb{R}^{d}$ be a bounded open domain and $L$ be a lattice of $\mathbb{R}^{d}$. Then $\Omega+L=\mathbb{R}^{d}$ if and only if $\left\{\mathrm{e}^{2 \pi i \alpha \cdot x}: \alpha \in L^{\perp}\right\}$ is an orthonormal basis with respect to the inner product (2.2).

The orthonormal property is defined with respect to normalized Lebesgue measure on $\Omega$. If $L=L_{A}$, then the measure of $\Omega$ is equal to $|\operatorname{det} A|$. Furthermore, since $L_{A}^{\perp}=A^{-\operatorname{tr}} \mathbb{Z}^{d}$, we can write $\alpha=A^{-\operatorname{tr}} k$ for $\alpha \in L_{A}^{\perp}$ and $k \in \mathbb{Z}^{d}$, so that $\alpha \cdot x=\left(A^{-\operatorname{tr}} k\right) \cdot x=k^{\operatorname{tr}} A^{-1} x$. Hence the orthogonality in the theorem is

$$
\frac{1}{|\operatorname{det}(A)|} \int_{\Omega} \mathrm{e}^{2 \pi i k^{\mathrm{tr}} A^{-1} \xi} d \xi=\delta_{k, 0}, \quad k \in \mathbb{Z}^{d}
$$

The set $\Omega$ is called a spectral (fundamental) set for the lattice $L$. If $L=L_{A}$ we also write $\Omega=\Omega_{A}$.

A function $f \in L^{1}\left(\Omega_{A}\right)$ can be expanded into a Fourier series

$$
f(x) \sim \sum_{k \in \mathbb{Z}^{d}} c_{k} \mathrm{e}^{2 \pi i k^{\mathrm{tr}} A^{-1} x}, \quad c_{k}=\frac{1}{|\operatorname{det}(A)|} \int_{\Omega} f(x) \mathrm{e}^{-2 \pi i k^{\mathrm{tr}} A^{-1} x} d x
$$

which is just a change of variables away from the usual multiple Fourier series. The Fourier transform $\widehat{f}$ of a function $f \in L^{1}\left(\mathbb{R}^{d}\right)$ and its inverse are defined by

$$
\widehat{f}(\xi)=\int_{\mathbb{R}^{d}} f(x) \mathrm{e}^{-2 \pi i \xi \cdot x} d x \quad \text { and } \quad f(x)=\int_{\mathbb{R}^{d}} \widehat{f}(\xi) \mathrm{e}^{2 \pi i \xi \cdot x} d \xi .
$$

One important tool for us is the Poisson summation formula given by

$$
\sum_{k \in \mathbb{Z}^{d}} f(x+A k)=\frac{1}{|\operatorname{det}(A)|} \sum_{k \in \mathbb{Z}^{d}} \widehat{f}\left(A^{-\mathrm{tr}} k\right) \mathrm{e}^{2 \pi i k^{\mathrm{tr}} A^{-1} x},
$$

which holds for $f \in L^{1}\left(\Omega_{A}\right)$ under the usual assumption on the convergence of the series in both sides. This formula has been used by various authors (see, for example, [1, 5, 7, 9, 19]). An immediate consequence of the Poisson summation formula is the following sampling theorem (see, for example, [7, 11]).

Proposition 2.2. Let $\Omega$ be a spectral set of the lattice $A \mathbb{Z}^{d}$. Assume that $\widehat{f}$ is supported on $\Omega$ and $\widehat{f} \in L^{2}(\Omega)$. Then

$$
f(x)=\sum_{k \in \mathbb{Z}^{d}} f\left(A^{-\operatorname{tr}} k\right) \Psi_{\Omega}\left(x-A^{-\operatorname{tr}} k\right)
$$

in $L^{2}(\Omega)$, where

$$
\Psi_{\Omega}(x)=\frac{1}{|\operatorname{det}(A)|} \int_{\Omega} \mathrm{e}^{2 \pi i x \cdot \xi} d \xi
$$

In fact, since $\widehat{f}$ is supported on $\Omega_{A}, \widehat{f}(\xi)=\sum_{k \in \mathbb{Z}^{d}} \widehat{f}(\xi+A k)$. Integrating over $\Omega$, the sampling equation follows from (2.4) and Fourier inversion. We note that

$$
\Psi_{\Omega}\left(A^{-\mathrm{tr}} j\right)=\delta_{0, j}, \quad \text { for all } j \in \mathbb{Z}^{d},
$$

by (2.3), so that $\Psi_{\Omega}$ can be considered as a cardinal interpolation function. 
2.2. Discrete Fourier analysis and interpolation. A function $f$ defined on $\mathbb{R}^{d}$ is called a periodic function with respect to the lattice $A \mathbb{Z}^{d}$ if

$$
f(x+A k)=f(x) \quad \text { for all } k \in \mathbb{Z}^{d} .
$$

For a given lattice $L$, its spectral set is evidently not unique. The orthogonality (2.3) is independent of the choice of $\Omega$. This can be seen directly from the fact that the function $x \mapsto e^{2 \pi i k^{\mathrm{tr}} A^{-1} x}$ is periodic with respect to the lattice $A \mathbb{Z}^{d}$.

In order to carry out a discrete Fourier analysis with respect to a lattice, we shall fix $\Omega$ such that $\Omega$ contains 0 in its interior and we further require in this subsection that the tiling $\Omega+A \mathbb{Z}^{d}=\mathbb{R}^{d}$ holds pointwise and without overlapping. In other words, we require

$$
\sum_{k \in \mathbb{Z}^{d}} \chi_{\Omega}(x+A k)=1, \forall x \in \mathbb{R}^{d}, \quad \text { and } \quad(\Omega+A k) \cap(\Omega+A j)=\emptyset, \text { if } k \neq j .
$$

For example, we can take $\Omega=\left[-\frac{1}{2}, \frac{1}{2}\right)^{d}$ for the standard lattice $\mathbb{Z}^{d}$.

Definition 2.3. Let $A$ and $B$ be two nonsingular matrices such that $\Omega_{A}$ and $\Omega_{B}$ satisfy (2.7). Assume that all entries of $N:=B^{\operatorname{tr}} A$ are integers. Denote

$$
\Lambda_{N}:=\left\{k \in \mathbb{Z}^{d}: B^{-\mathrm{tr}} k \in \Omega_{A}\right\}, \quad \text { and } \quad \Lambda_{N_{\mathrm{tr}}}:=\left\{k \in \mathbb{Z}^{d}: A^{-\mathrm{tr}} k \in \Omega_{B}\right\} .
$$

Two points $x, y \in \mathbb{R}^{d}$ are said to be congruent with respect to the lattice $A \mathbb{Z}^{d}$, if $x-y \in A \mathbb{Z}^{d}$, and we write $x \equiv y \bmod A$.

Theorem 2.4. Let $A, B$ and $N$ be as in Definition 2.3. Then

$$
\frac{1}{|\operatorname{det}(N)|} \sum_{j \in \Lambda_{N}} \mathrm{e}^{2 \pi i k^{\mathrm{tr}} N^{-1} j}= \begin{cases}1, & \text { if } k \equiv 0 \quad \bmod N^{\mathrm{tr}}, \\ 0, & \text { otherwise. }\end{cases}
$$

Proof. Let $f \in L^{1}\left(\mathbb{R}^{d}\right)$ be such that Poisson summation formula holds. Denote

$$
\Phi^{A} f(x):=\sum_{k \in \mathbb{Z}^{d}} f(x+A k) .
$$

Then the Poisson summation formula (2.4) shows that

$$
\Phi^{A} f\left(B^{-\operatorname{tr}} j\right)=\frac{1}{|\operatorname{det}(A)|} \sum_{k \in \mathbb{Z}^{d}} \widehat{f}\left(A^{-\mathrm{tr}} k\right) e^{2 \pi i k^{\mathrm{tr}} N^{-1} j}, \quad j \in \mathbb{Z}^{d} .
$$

Since $\mathbb{R}^{d}=\Omega_{B}+B \mathbb{Z}^{d}$, we can write $A^{- \text {tr }} k=x_{B}+B m$ for every $k \in \mathbb{Z}^{d}$ with $x_{B} \in \Omega_{B}$ and $m \in \mathbb{Z}^{d}$. The fact that $N$ has integer entries shows that $A^{\mathrm{tr}} x_{B}=$ $l \in \mathbb{Z}^{d}$. That is, every $k \in \mathbb{Z}^{d}$ can be written as $k=l+N^{\operatorname{tr}} m$ with $l \in \Lambda_{N^{\text {tr }}}$ and $m \in \mathbb{Z}^{d}$. It is easy to see that $\mathrm{e}^{2 \pi i k^{\mathrm{tr}} N^{-1} j}=\mathrm{e}^{2 \pi i l^{\mathrm{tr}} N^{-1} j}$ under this decomposition. Consequently,

$$
\begin{aligned}
\Phi^{A} f\left(B^{-\mathrm{tr}} j\right) & =\frac{1}{|\operatorname{det}(A)|} \sum_{l \in \Lambda_{N} \operatorname{tr}} \sum_{m \in \mathbb{Z}^{d}} \widehat{f}\left(A^{-\mathrm{tr}} l+B m\right) \mathrm{e}^{2 \pi i l^{\mathrm{tr}} N^{-1} j} \\
& =\frac{1}{|\operatorname{det}(A)|} \sum_{l \in \Lambda_{N} \operatorname{tr}} \Phi^{B} \widehat{f}\left(A^{-\mathrm{tr}} l\right) \mathrm{e}^{2 \pi i l^{\mathrm{tr}} N^{-1} j} .
\end{aligned}
$$

Similarly, we have

$$
\Phi^{B} \widehat{f}\left(A^{-\mathrm{tr}} l\right)=\frac{1}{|\operatorname{det}(B)|} \sum_{j \in \Lambda_{N}} \Phi^{A} f\left(B^{-\mathrm{tr}} j\right) \mathrm{e}^{-2 \pi i l^{\mathrm{tr}} N^{-1} j} .
$$


Substituting (2.9) into (2.10) leads to the identity

$$
\Phi^{B} \widehat{f}\left(A^{-\mathrm{tr}} l\right)=\frac{1}{|\operatorname{det}(A B)|} \sum_{k \in \Lambda_{N}^{\mathrm{tr}}} \Phi^{B} \widehat{f}\left(A^{-\mathrm{tr}} k\right) \sum_{j \in \Lambda_{N}} \mathrm{e}^{2 \pi i(k-l)^{\mathrm{tr}} N^{-1} j},
$$

that holds for $f$ in, say, the Schwartz class of functions, from which (2.8) follows immediately.

Theorem 2.5. Let $A, B$ and $N$ be as in Definition 2.3. Define

$$
\langle f, g\rangle_{N}=\frac{1}{|\operatorname{det}(N)|} \sum_{j \in \Lambda_{N}} f\left(B^{-\operatorname{tr}} j\right) \overline{g\left(B^{-\operatorname{tr}} j\right)}
$$

for $f, g$ in $C\left(\Omega_{A}\right)$, the space of continuous functions on $\Omega_{A}$. Then

$$
\langle f, g\rangle_{\Omega_{A}}=\langle f, g\rangle_{N}
$$

for all $f, g$ in the finite dimensional subspace

$$
\mathcal{H}_{N}:=\operatorname{span}\left\{\phi_{k}: \phi_{k}(x)=\mathrm{e}^{2 \pi i k^{\mathrm{tr}} A^{-1} x}, k \in \Lambda_{N^{\mathrm{tr}}}\right\} .
$$

Proof. By the definition of $\langle\cdot, \cdot\rangle_{N}$, equation (2.8) implies that $\left\langle\phi_{l}, \phi_{k}\right\rangle_{N}=\delta_{l, k}$, $l, k \in N^{\mathrm{tr}}$, which shows, by (2.3), that (2.11) holds for $f=\phi_{l}$ and $g=\phi_{k}$. Since both inner products are sesquilinear, the stated result follows.

If $\Lambda$ is a subset of $\mathbb{Z}^{d}$, we denote by $\# \Lambda$ the number of points in $\Lambda$. Evidently the dimension of $\mathcal{H}_{N}$ is $\# \Lambda_{N^{\mathrm{tr}}}$.

Let $\mathcal{I}_{N} f$ denote the Fourier expansion of $f \in C\left(\Omega_{A}\right)$ in $\mathcal{H}_{N}$ with respect to the inner product $\langle\cdot, \cdot\rangle$. Then

$$
\begin{aligned}
\mathcal{I}_{N} f(x) & :=\sum_{j \in \Lambda_{N} \mathrm{tr}}\left\langle f, \phi_{j}\right\rangle_{N} \phi_{j}(x) \\
& =\frac{1}{|\operatorname{det}(N)|} \sum_{k \in \Lambda_{N}} f\left(B^{-\operatorname{tr}} k\right) \sum_{j \in \Lambda_{N} \text { tr }} \phi_{j}(x) \overline{\phi_{j}\left(B^{-\operatorname{tr}} k\right)} .
\end{aligned}
$$

Hence, analogous to the sampling theorem in Proposition 2.2 we have

$$
\mathcal{I}_{N} f(x)=\sum_{k \in \Lambda_{N}} f\left(B^{-\operatorname{tr}} k\right) \Psi_{\Omega_{B}}^{A}\left(x-B^{-\mathrm{tr}} k\right), \quad f \in C\left(\Omega_{A}\right),
$$

where

$$
\Psi_{\Omega_{B}}^{A}(x)=\frac{1}{|\operatorname{det}(N)|} \sum_{j \in \Lambda_{N} \operatorname{tr}} \mathrm{e}^{2 \pi i j^{\mathrm{tr}} A^{-1} x} .
$$

Theorem 2.6. Let $A, B$ and $N$ be as in Definition 2.3. Then $\mathcal{I}_{N} f$ is the unique interpolation operator on $N$ in $\mathcal{H}_{N}$; that is,

$$
\mathcal{I}_{N} f\left(B^{-\operatorname{tr}} j\right)=f\left(B^{-\operatorname{tr}} j\right), \quad \forall j \in \Lambda_{N} .
$$

In particular, $\# \Lambda_{N}=\# \Lambda_{N^{\mathrm{tr}}}=|\operatorname{det}(N)|$. Furthermore, the fundamental interpolation function $\Psi_{\Omega_{A}}^{B}$ satisfies

$$
\Psi_{\Omega_{B}}^{A}(x)=\sum_{j \in \mathbb{Z}^{d}} \Psi_{\Omega_{B}}(x+A j) .
$$


Proof. Using (2.8) with $N$ in place of $N^{\text {tr }}$ gives immediately that $\Psi_{\Omega_{B}}^{A}\left(B^{-\operatorname{tr}} k\right)=\delta_{k, 0}$ for $k \in \Lambda_{N}$, so that the interpolation holds. This also shows that $\left\{\Psi_{\Omega_{B}}^{A}(x-\right.$ $\left.\left.B^{-\operatorname{tr}} k\right): k \in \Lambda_{N}\right\}$ is linearly independent. The equation (2.8) with $k=0$ shows immediately that $\# \Lambda_{N}=|\operatorname{det}(N)|$. Similarly, with $N$ replaced by $N^{\mathrm{tr}}$, we have $\# \Lambda_{N}=\left|\operatorname{det}\left(N^{\mathrm{tr}}\right)\right|$. This shows that $\# \Lambda_{N}=|\operatorname{det}(N)|=\# \Lambda_{N^{\mathrm{tr}}}$. Furthermore, let $M=\left(\phi_{k}\left(B^{-\operatorname{tr}} j\right)\right)_{j, k \in \Lambda_{N}}$ be the interpolation matrix, then the equation (2.8) shows that $M$ is a unitary matrix, which shows in particular that $M$ is invertible. Consequently the interpolation by $\mathcal{H}_{n}$ on points in $\Lambda_{N}$ is unique.

Finally, since $\widehat{\Psi}_{\Omega_{B}}=|\operatorname{det}(B)|^{-1} \chi_{\Omega_{B}}$, we have

$$
\begin{aligned}
\Psi_{\Omega_{B}}^{A}(x) & =\frac{1}{|\operatorname{det}(N)|} \sum_{j \in \mathbb{Z}^{d}} \phi_{j}(x) \chi_{\Omega_{B}}\left(A^{-\operatorname{tr}} j\right) \\
& =\frac{1}{|\operatorname{det}(A)|} \sum_{j \in \mathbb{Z}^{d}} \phi_{j}(x) \widehat{\Psi}_{\Omega_{B}}\left(A^{-\operatorname{tr}} j\right),
\end{aligned}
$$

from which (2.14) follows from the Poisson summation formula (2.4).

The result in this section applies to fairly general lattices. In the following section we apply it to the hexagon lattice in two dimension. We hope to report results for higher dimensional lattices in a future work.

\section{Discrete Fourier analysis on the HeXagon}

3.1. Hexagon lattice and Fourier analysis. The generator matrix and the spectral set of the hexagon lattice is given by

$$
H=\left(\begin{array}{cc}
\sqrt{3} & 0 \\
-1 & 2
\end{array}\right), \quad \Omega_{H}=\left\{\left(x_{1}, x_{2}\right):-1 \leq x_{2}, \frac{\sqrt{3}}{2} x_{1} \pm \frac{1}{2} x_{2}<1\right\} .
$$

The strict inequality in the definition of $\Omega_{H}$ comes from our assumption in (2.7).

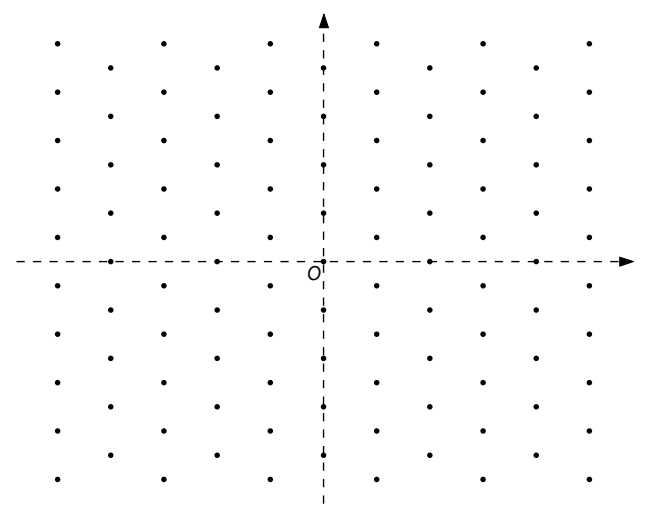

Figure 1. The lattice $L_{H}$.

As shown in [21], it is more convenient to use homogeneous coordinates $\left(t_{1}, t_{2}, t_{3}\right)$ that satisfies $t_{1}+t_{2}+t_{3}=0$. We define

$$
t_{1}=-\frac{x_{2}}{2}+\frac{\sqrt{3} x_{1}}{2}, \quad t_{2}=x_{2}, \quad t_{3}=-\frac{x_{2}}{2}-\frac{\sqrt{3} x_{1}}{2} .
$$


Under these homogeneous coordinates, the hexagon $\Omega_{H}$ becomes

$$
\Omega=\left\{\left(t_{1}, t_{2}, t_{3}\right) \in \mathbb{R}^{3}:-1 \leq t_{1}, t_{2},-t_{3}<1 ; t_{1}+t_{2}+t_{3}=0\right\},
$$

which is the intersection of the plane $t_{1}+t_{2}+t_{3}=0$ with the cube $[-1,1]^{3}$, as shown in Figure 2. Later in the paper we shall depict the hexagon as a two dimensional figure, but still refer to its points by their homogeneous coordinates, as shown by the right hand side figure of Figure 2.
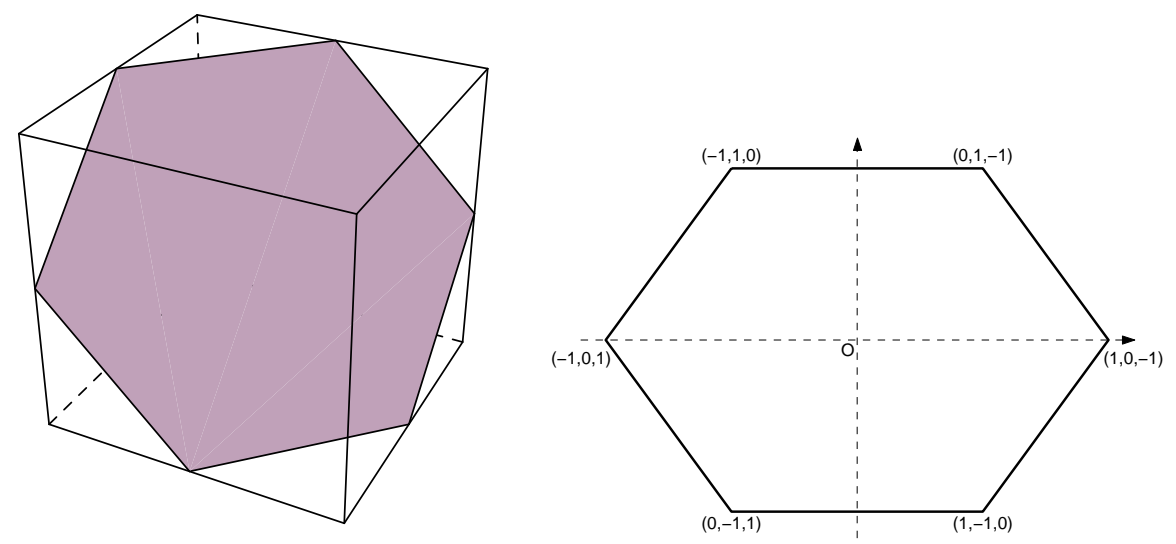

Figure 2. The hexagon in homogeneous coordinates.

For convenience, we adopt the convention of using bold letters, such as $\mathbf{t}$, to denote points in the space

$$
\mathbb{R}_{H}^{3}:=\left\{\mathbf{t}=\left(t_{1}, t_{2}, t_{3}\right) \in \mathbb{R}^{3}: t_{1}+t_{2}+t_{3}=0\right\} .
$$

In other words, bold letters such as $\mathbf{t}$ stand for homogeneous coordinates. Furthermore, we introduce the notation

$$
\mathbb{H}:=\mathbb{Z}^{3} \cap \mathbb{R}_{H}^{3}=\left\{\mathbf{k}=\left(k_{1}, k_{2}, k_{3}\right) \in \mathbb{Z}^{3}: k_{1}+k_{2}+k_{3}=0\right\} .
$$

The inner product on the hexagon under homogeneous coordinates becomes

$$
\langle f, g\rangle_{H}=\frac{1}{\left|\Omega_{H}\right|} \int_{\Omega_{H}} f\left(x_{1}, x_{2}\right) \overline{g\left(x_{1}, x_{2}\right)} d x_{1} d x_{2}=\frac{1}{|\Omega|} \int_{\Omega} f(\mathbf{t}) \overline{g(\mathbf{t})} d \mathbf{t},
$$

where $|\Omega|$ denotes the area of $\Omega$.

To apply the general results in the previous section, we choose $A=H$ and $B=\frac{n}{2} H$, where $n$ is a positive integer. Then

$$
N=B^{\operatorname{tr}} A=\frac{n}{2} H^{T} H=\left[\begin{array}{cc}
2 n & -n \\
-n & 2 n
\end{array}\right]
$$

has integer entries. Note that $N$ is a symmetric matrix so that $\Lambda_{N}=\Lambda_{N^{\mathrm{tr}}}$. Using the fact that $B^{-\operatorname{tr}} j=\frac{2}{n} H^{-\operatorname{tr}} j=\frac{1}{n}\left(\frac{2}{\sqrt{3}} j_{1}+\frac{1}{\sqrt{3}} j_{2}, j_{2}\right)$, it is not hard to see that $B^{-\operatorname{tr}} j \in \Omega_{A}$, or equivalently $j \in \Lambda_{N}$, becomes $\mathbf{j}=\left(j_{1}, j_{2},-j_{1}-j_{2}\right) \in \mathbb{H}_{n}$, where

$$
\mathbb{H}_{n}:=\left\{\mathbf{j} \in \mathbb{H}:-n \leq j_{1}, j_{2},-j_{3}<n\right\} \quad \text { and } \quad \# \mathbb{H}_{n}=3 n^{2} .
$$


In other words, $\Lambda_{N}$ in the previous section becomes $\mathbb{H}_{n}$ in homogeneous coordinates. The fact that $\# \mathbb{H}_{n}=3 n^{2}$ can be easily verified. Moreover, under the change of variables $x=\left(x_{1}, x_{2}\right) \mapsto\left(t_{1}, t_{2}, t_{3}\right)$ in (3.1), it is easy to see that

$$
\left(k_{1}, k_{2}\right) H^{-1} x=\frac{1}{3}\left(k_{1}, k_{2},-k_{1}-k_{2}\right) \mathbf{t}=\frac{1}{3} \mathbf{k} \cdot \mathbf{t},
$$

so that $\mathrm{e}^{2 \pi i k^{\mathrm{tr}} H^{-1} x}=\mathrm{e}^{\frac{2 \pi i}{3} \mathbf{k}^{\mathrm{tr}} \mathbf{t}}$. Therefore, introducing the notation

$$
\phi_{\mathbf{j}}(\mathbf{t}):=\mathrm{e}^{\frac{2 \pi i}{3} \mathbf{j}^{\mathrm{t}} \mathbf{t}}, \quad \mathbf{j} \in \mathbb{H},
$$

the orthogonality relation (2.3) becomes

$$
\left\langle\phi_{\mathbf{k}}, \phi_{\mathbf{j}}\right\rangle_{H}=\delta_{\mathbf{k}, \mathbf{j}}, \quad \mathbf{k}, \mathbf{j} \in \mathbb{H} .
$$

The finite dimensional space $\mathcal{H}_{N}$ in the previous section becomes

$$
\mathcal{H}_{n}:=\operatorname{span}\left\{\phi_{\mathbf{j}}: \mathbf{j} \in \mathbb{H}_{n}\right\} \text {, and } \operatorname{dim} \mathcal{H}_{n}=3 n^{2} .
$$

Under the homogeneous coordinates (3.1), $x \equiv y(\bmod H)$ becomes $\mathbf{t} \equiv \mathbf{s}$ $\bmod 3$, where we define

$$
\mathbf{t} \equiv \mathbf{s} \bmod 3 \quad \Longleftrightarrow \quad t_{1}-s_{1} \equiv t_{2}-s_{2} \equiv t_{3}-s_{3} \quad \bmod 3 .
$$

We call a function $f$ H-periodic if $f(\mathbf{t})=f(\mathbf{t}+\mathbf{j})$ whenever $\mathbf{j} \equiv 0 \bmod 3$. Since $\mathbf{j}, \mathbf{k} \in \mathbb{H}$ implies that $2 \mathbf{j} \cdot \mathbf{k}=\left(j_{1}-j_{2}\right)\left(k_{1}-k_{2}\right)+3 j_{3} k_{3}$, we see that $\phi_{\mathbf{j}}$ is H-periodic. The following lemma is convenient in practice.

Lemma 3.1. Let $\varepsilon_{1}=(2,-1,-1), \varepsilon_{2}=(-1,2,-1), \varepsilon_{3}=(-1,-1,2)$. Then $a$ function $f(\mathbf{t})$ is H-periodic if and only if

$$
f\left(\mathbf{t}+j \varepsilon_{i}\right)=f(\mathbf{t}), \quad j \in \mathbb{Z}, \quad i=1,2,3 .
$$

3.2. Discrete Fourier analysis on the regular hexagon. Using the set-up in the previous subsection, Theorem 2.5 becomes, in homogeneous coordinates, the following:

Proposition 3.2. For $n \geq 0$, define

$$
\langle f, g\rangle_{n}:=\frac{1}{3 n^{2}} \sum_{\mathbf{j} \in \mathbb{H}_{n}} f\left(\frac{\mathbf{j}}{n}\right) \overline{g\left(\frac{\mathbf{j}}{n}\right)}, \quad f, g \in C(\Omega) .
$$

Then

$$
\langle f, g\rangle_{H}=\langle f, g\rangle_{n}, \quad f, g \in \mathcal{H}_{n} .
$$

The point set $\mathbb{H}_{n}$, hence the inner product $\langle\cdot, \cdot\rangle_{n}$, is not symmetric on $\Omega$ in the sense that it contains only part of the points on the boundary, as shown by the left hand figure in Figure 3. We denote by $\mathbb{H}_{n}^{*}$ the symmetric point set on $\Omega$,

$$
\mathbb{H}_{n}^{*}:=\left\{\mathbf{j} \in \mathbb{H}:-n \leq j_{1}, j_{2}, j_{3} \leq n\right\}, \quad \text { and } \quad \# \mathbb{H}_{n}^{*}=3 n^{2}+3 n+1 .
$$

The set $\mathbb{H}_{n}^{*}$ is shown by the right hand figure in Figure 3.

Using periodicity, we can show that the inner product $\langle\cdot, \cdot\rangle$ is equivalent to a symmetric discrete inner product based on $\mathbb{H}_{n}^{*}$. Let

$$
\mathbb{H}_{n}^{\circ}:=\left\{\mathbf{j} \in \mathbb{H}:-n<j_{1}, j_{2}, j_{3}<n\right\}
$$

denote the set of interior points in $\mathbb{H}_{n}^{*}$ and $\mathbb{H}_{n}$. Define

$$
\langle f, g\rangle_{n}^{*}:=\frac{1}{3 n^{2}} \sum_{\mathbf{j} \in \mathbb{H}_{n}^{*}} c_{\mathbf{j}}^{(n)} f\left(\frac{\mathbf{j}}{n}\right) \overline{g\left(\frac{\mathbf{j}}{n}\right)}, \quad f, g \in C(\Omega),
$$



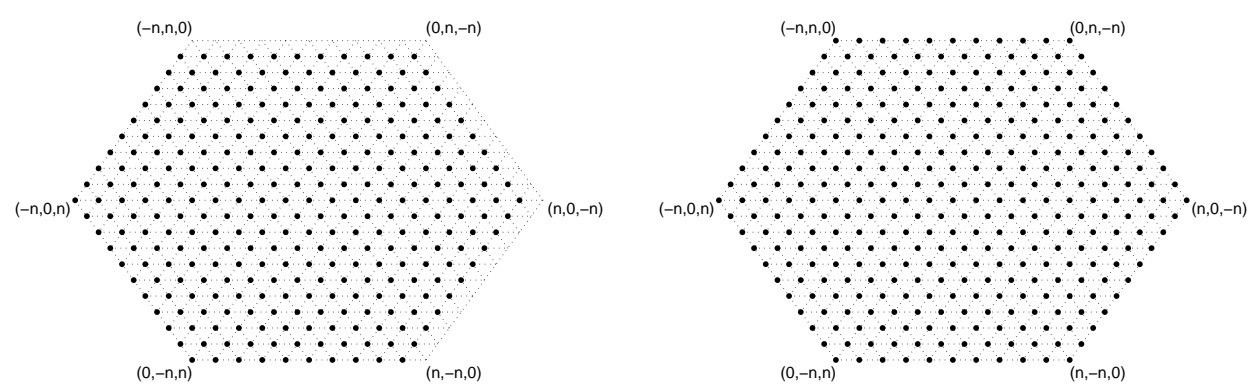

FigurE 3. The set $\mathbb{H}_{n}$ and the set $\mathbb{H}_{n}^{*}$.

where

$$
c_{\mathbf{j}}^{(n)}= \begin{cases}1, & \mathbf{j} \in \mathbb{H}_{n}^{\circ}, \quad \text { (inner points), } \\ \frac{1}{2}, & \mathbf{j} \in \mathbb{H}_{n}^{*} \backslash \mathbb{H}_{n}^{\circ}, j_{1} j_{2} j_{3} \neq 0, \quad \text { (edge points) }, \\ \frac{1}{3}, & \mathbf{j} \in \mathbb{H}_{n}^{*} \backslash \mathbb{H}_{n}^{\circ}, j_{1} j_{2} j_{3}=0, \quad \text { (vertices) },\end{cases}
$$

in which we also include the positions of the points; the only one that needs an explanation is the "edge points", which are the points located on the edges but not on the vertices of the hexagon. In analogy to Proposition 3.2, we have the following theorem.

Theorem 3.3. For $n \geq 0$,

$$
\langle f, g\rangle_{H}=\langle f, g\rangle_{n}=\langle f, g\rangle_{n}^{*}, \quad f, g \in \mathcal{H}_{n} .
$$

Proof. If $f$ is an H-periodic function, then it follows from (3.4) that

$$
\begin{aligned}
6 \sum_{\mathbf{j} \in \mathbb{H}_{n}^{*} \backslash \mathbb{H}_{n}} c_{\mathbf{j}}^{(n)} f\left(\frac{\mathbf{j}}{n}\right)=2[f(1,-1,0)+f(1,0,-1)+f(0,1,-1)+f(-1,1,0)] \\
\quad+3 \sum_{1 \leq j \leq n-1}\left[f\left(1,-\frac{j}{n}, \frac{j}{n}-1\right)+f\left(\frac{j}{n}-1,1,-\frac{j}{n}\right)+f\left(1-\frac{j}{n}, \frac{j}{n},-1\right)\right] \\
=3 \sum_{1 \leq j \leq n-1}\left[f\left(-1,1-\frac{j}{n}, \frac{j}{n}\right)+f\left(\frac{j}{n},-1,1-\frac{j}{n}\right)+f\left(-\frac{j}{n}, \frac{j}{n}-1,1\right)\right] \\
\quad+4[f(0,-1,1)+f(-1,0,1)] \\
=6 \sum_{\mathbf{j} \in \mathbb{H}_{n} \backslash \mathbb{H}_{n-1}^{*}}\left(1-c_{\mathbf{j}}^{(n)}\right) f\left(\frac{\mathbf{j}}{n}\right)=6 \sum_{\mathbf{j} \in \mathbb{H}_{n}}\left(1-c_{\mathbf{j}}^{(n)}\right) f\left(\frac{\mathbf{j}}{n}\right) .
\end{aligned}
$$

Consequently, we have

$$
\begin{aligned}
\sum_{\mathbf{j} \in \mathbb{H}_{n}^{*}} c_{\mathbf{j}}^{(n)} f\left(\frac{\mathbf{j}}{n}\right) & =\sum_{\mathbf{j} \in \mathbb{H}_{n}} c_{\mathbf{j}}^{(n)} f\left(\frac{\mathbf{j}}{n}\right)+\sum_{\mathbf{j} \in \mathbb{H}_{n}^{*} \backslash \mathbb{H}_{n}} c_{\mathbf{j}}^{(n)} f\left(\frac{\mathbf{j}}{n}\right) \\
& =\sum_{\mathbf{j} \in \mathbb{H}_{n}} c_{\mathbf{j}}^{(n)} f\left(\frac{\mathbf{j}}{n}\right)+\sum_{\mathbf{j} \in \mathbb{H}_{n}}\left(1-c_{\mathbf{j}}^{(n)}\right) f\left(\frac{\mathbf{j}}{n}\right)=\sum_{\mathbf{j} \in \mathbb{H}_{n}} f\left(\frac{\mathbf{j}}{n}\right) .
\end{aligned}
$$

Replacing $f$ by $f \bar{g}$, we have proved that $\langle f, g\rangle_{n}=\langle f, g\rangle_{n}^{*}$ for $H$-periodic functions, which clearly applies to functions in $\mathcal{H}_{n}$. 
3.3. Interpolation on the hexagon. First we note that by (3.2), Theorem 2.6 when restricted to the hexagon domain becomes the following:

Proposition 3.4. For $n \geq 0$, define

$$
\mathcal{I}_{n} f(\mathbf{t}):=\sum_{\mathbf{j} \in \mathbb{H}_{n}} f\left(\frac{\mathbf{j}}{n}\right) \Phi_{n}\left(\mathbf{t}-\frac{\mathbf{j}}{n}\right), \quad \text { where } \quad \Phi_{n}(\mathbf{t})=\frac{1}{3 n^{2}} \sum_{\mathbf{j} \in \mathbb{H}_{n}} \phi_{\mathbf{j}}(\mathbf{t}),
$$

for $f \in C(\Omega)$. Then $\mathcal{I}_{n} f \in \mathcal{H}_{n}$ and

$$
\mathcal{I}_{n} f\left(\frac{\mathbf{j}}{n}\right)=f\left(\frac{\mathbf{j}}{n}\right), \quad \forall \mathbf{j} \in \mathbb{H}_{n} .
$$

Furthermore, and perhaps much more interesting, is another interpolation operator that works for almost all points in $\mathbb{H}_{n}^{*}$. First we need a few more definitions. We denote by $\partial \mathbb{H}_{n}^{*}:=\mathbb{H}_{n}^{*} \backslash \mathbb{H}_{n}^{\circ}$, the set of points in $\mathbb{H}_{n}^{*}$ that are on the boundary of the hexagon $\left\{\mathbf{t}:-n \leq t_{1}, t_{2}, t_{3} \leq n\right\}$. We further divide $\partial \mathbb{H}_{n}^{*}$ as $\mathbb{H}_{n}^{\mathrm{v}} \cup \mathbb{H}_{n}^{\mathrm{e}}$, where $\mathbb{H}_{n}^{\mathrm{v}}$ consists of the six vertices of $\partial \mathbb{H}_{n}$ and $\mathbb{H}_{n}^{\mathrm{e}}$ consists of the other points in $\partial \mathbb{H}_{n}$, i.e., the edge points. For $\mathbf{j} \in \partial \mathbb{H}_{n}^{*}$, we define

$$
\mathcal{S}_{\mathbf{j}}:=\left\{\mathbf{k} \in \mathbb{H}_{n}^{*}: \frac{\mathbf{k}}{n} \equiv \frac{\mathbf{j}}{n} \bmod 3\right\} .
$$

Because of the tiling property of the hexagon, if $\mathbf{j} \in \mathbb{H}_{n}^{\mathrm{e}}$ then $\mathcal{S}_{\mathbf{j}}$ contains two points, $\mathbf{j}$ and $\mathbf{j}^{*} \in \mathbb{H}_{n}^{\mathrm{e}}$, where $j^{*}$ is on the opposite edge, relative to $j$, of the hexagon; while if $\mathbf{j} \in \mathbb{H}_{n}^{\mathrm{v}}$, then $\mathcal{S}_{\mathbf{j}}$ contains three vertices, $\mathbf{j}$ and its rotations by the angles $2 \pi / 3$ and $4 \pi / 3$.

Theorem 3.5. For $n \geq 0$ and $f \in C(\Omega)$, define

$$
\mathcal{I}_{n}^{*} f(\mathbf{t}):=\sum_{\mathbf{j} \in \mathbb{H}_{n}^{*}} f\left(\frac{\mathbf{j}}{n}\right) \ell_{\mathbf{j}, n}(\mathbf{t})
$$

where

$$
\ell_{\mathbf{j}, n}(\mathbf{t})=\Phi_{n}^{*}\left(\mathbf{t}-\frac{\mathbf{j}}{n}\right) \quad \text { and } \quad \Phi_{n}^{*}(\mathbf{t})=\frac{1}{3 n^{2}} \sum_{\mathbf{j} \in \mathbb{H}_{n}^{*}} c_{\mathbf{j}}^{(n)} \phi_{\mathbf{j}}(\mathbf{t}) .
$$

Then $\mathcal{I}_{n}^{*} f \in \mathcal{H}_{n}^{*}:=\left\{\phi_{\mathbf{j}}: \mathbf{j} \in \mathbb{H}_{n}^{*}\right\}$ and

$$
\mathcal{I}_{n}^{*} f\left(\frac{\mathbf{j}}{n}\right)= \begin{cases}f\left(\frac{\mathbf{j}}{n}\right), & \mathbf{j} \in \mathbb{H}_{n}^{\circ}, \\ \sum_{\mathbf{k} \in \mathcal{S}_{\mathbf{j}}} f\left(\frac{\mathbf{k}}{n}\right), & \mathbf{j} \in \partial \mathbb{H}_{n}^{*}\end{cases}
$$

Furthermore, $\Phi_{n}^{*}(\mathbf{t})$ is a real function and it has a compact formula

$$
\begin{aligned}
\Phi_{n}^{*}(\mathbf{t})=\frac{1}{3 n^{2}}[ & \frac{1}{2}\left(\Theta_{n}(\mathbf{t})-\Theta_{n-2}(\mathbf{t})\right) \\
& \left.-\frac{1}{3}\left(\cos \frac{2 n \pi}{3}\left(t_{1}-t_{2}\right)+\cos \frac{2 n \pi}{3}\left(t_{2}-t_{3}\right)+\cos \frac{2 n \pi}{3}\left(t_{3}-t_{1}\right)\right)\right]
\end{aligned}
$$

where

$$
\Theta_{n}(\mathbf{t}):=\frac{\sin \frac{(n+1) \pi\left(t_{1}-t_{2}\right)}{3} \sin \frac{(n+1) \pi\left(t_{2}-t_{3}\right)}{3} \sin \frac{(n+1) \pi\left(t_{3}-t_{1}\right)}{3}}{\sin \frac{\pi\left(t_{1}-t_{2}\right)}{3} \sin \frac{\pi\left(t_{2}-t_{3}\right)}{3} \sin \frac{\pi\left(t_{3}-t_{1}\right)}{3}} .
$$

Proof. First let $\mathbf{k} \in \mathbb{H}_{n}$. From the definition of the inner product $\langle\cdot, \cdot\rangle_{n}^{*}$, it follows that if $\mathbf{j} \in \mathbb{H}_{n}^{\circ}$, then $\ell_{\mathbf{j}, n}\left(\frac{\mathbf{k}}{n}\right)=\left\langle\phi_{\mathbf{k}}, \phi_{\mathbf{j}}\right\rangle_{n}^{*}=\delta_{\mathbf{k}, \mathbf{j}}$. If $\mathbf{j} \in \partial \mathbb{H}_{n}^{*}$, then one of the points in 
$\mathcal{S}_{\mathbf{j}}$ will be in $\mathbb{H}_{n}$, call it $\mathbf{j}^{*}$, then $\ell_{\mathbf{j}, n}\left(\frac{\mathbf{k}}{n}\right)=\delta_{\mathbf{k}, \mathbf{j}^{*}}$. As the role of $\mathbf{j}$ and $\mathbf{k}$ is symmetric in $\ell_{\mathbf{j}, n}\left(\frac{\mathbf{k}}{n}\right)$, this covers all cases. Putting these cases together, we have proved that

$$
\ell_{\mathbf{j}, n}\left(\frac{\mathbf{k}}{n}\right)=\Phi_{n}^{*}\left(\frac{\mathbf{k}}{n}-\frac{\mathbf{j}}{n}\right)=\left\{\begin{array}{ll}
1 & \text { if } \frac{\mathbf{k}}{n} \equiv \frac{\mathbf{j}}{n} \quad \bmod 3 \\
0 & \text { otherwise }
\end{array}, \quad \mathbf{k}, \mathbf{j} \in \mathbb{H}_{n}^{*},\right.
$$

from which the interpolation (3.8) follows immediately.

By the definition of $c_{\mathbf{j}}^{(m)}$, it is easy to see that

$$
\Phi_{n}^{*}(\mathbf{t})=\frac{1}{3 n^{2}}\left[\frac{1}{2}\left(D_{n}(\mathbf{t})+D_{n-1}(\mathbf{t})\right)-\frac{1}{6} \sum_{\mathbf{j} \in \mathbb{H}_{n}^{v}} \phi_{\mathbf{j}}(\mathbf{t})\right],
$$

where $D_{n}$ is the analogue of the Dirichlet kernel defined by

$$
D_{n}(\mathbf{t}):=\sum_{\mathbf{j} \in \mathbb{H}_{n}^{*}} \phi_{\mathbf{j}}(\mathbf{t}) .
$$

By the definitions of $\phi_{\mathbf{k}}$ and $\mathbb{H}_{n}^{\mathrm{v}}$, we have

$$
\sum_{\mathbf{j} \in \mathbb{H}_{n}^{v}} \phi_{\mathbf{j}}(\mathbf{t})=2\left[\cos \frac{2 n \pi}{3}\left(t_{1}-t_{2}\right)+\cos \frac{2 n \pi}{3}\left(t_{2}-t_{3}\right)+\cos \frac{2 n \pi}{3}\left(t_{3}-t_{1}\right)\right],
$$

so that we only have to show that $D_{n}=\Theta_{n}-\Theta_{n-1}$, which in fact has already appeared in [21]. Since an intermediate step is needed later, we outline the proof of the identity below. The hexagon domain can be partitioned into three parallelograms as shown in Figure 4.

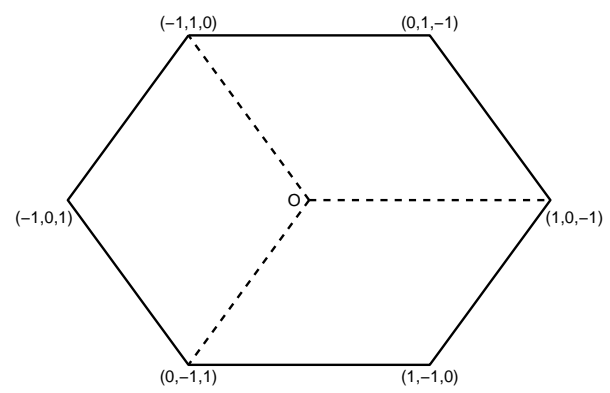

Figure 4. The hexagon partitioned into three parallelograms.

Accordingly, we split the sum over $\mathbb{H}_{n}^{*}$ as

$$
D_{n}(\mathbf{t})=1+\sum_{j_{2}=-n}^{-1} \sum_{j_{1}=0}^{n} \phi_{\mathbf{j}}(\mathbf{t})+\sum_{j_{3}=-n}^{-1} \sum_{j_{2}=0}^{n} \phi_{\mathbf{j}}(\mathbf{t})+\sum_{j_{1}=-n}^{-1} \sum_{j_{3}=0}^{n} \phi_{\mathbf{j}}(\mathbf{t}) .
$$

Using the fact that $t_{1}+t_{2}+t_{3}=0$ and $j_{1}+j_{2}+j_{3}=0$, each of the three sums can be summed up explicitly. For example,

$$
\begin{aligned}
& \sum_{j_{2}=-n}^{-1} \sum_{j_{1}=0}^{n} \phi_{\mathbf{j}}(\mathbf{t}) \\
& \quad=\frac{\mathrm{e}^{-\frac{\pi i}{3}(n+1)\left(t_{3}-t_{2}\right)} \sin \frac{n \pi}{3}\left(t_{2}-t_{3}\right)}{\sin \frac{\pi}{3}\left(t_{2}-t_{3}\right)} \frac{\mathrm{e}^{-\frac{\pi i}{3} n\left(t_{1}-t_{3}\right)} \sin \frac{(n+1) \pi}{3}\left(t_{1}-t_{3}\right)}{\sin \frac{\pi}{3}\left(t_{1}-t_{3}\right)} .
\end{aligned}
$$


The formula $D_{n}=\Theta_{n}-\Theta_{n-1}$ comes from summing up these terms and carrying out simplification using the fact that if $t_{1}+t_{2}+t_{3}=0$, then

$$
\begin{aligned}
\sin 2 t_{1}+\sin 2 t_{2}+\sin 2 t_{3} & =-4 \sin t_{1} \sin t_{2} \sin t_{3}, \\
\cos 2 t_{1}+\cos 2 t_{2}+\cos 2 t_{3} & =4 \cos t_{1} \cos t_{2} \cos t_{3}-1,
\end{aligned}
$$

which can be easily verified.

The compact formula of the interpolation function allows us to estimate the operator norm of $\mathcal{I}_{n}^{*}: C(\bar{\Omega}) \mapsto C(\bar{\Omega})$ in the uniform norm, which is often referred to as the Lebesgue constant.

Theorem 3.6. Let $\left\|\mathcal{I}_{n}^{*}\right\|_{\infty}$ denote the operator norm of $\mathcal{I}_{n}^{*}: C(\bar{\Omega}) \mapsto C(\bar{\Omega})$. Then there is a constant $c$, independent of $n$, such that

$$
\left\|\mathcal{I}_{n}^{*}\right\|_{\infty} \leq c(\log n)^{2} .
$$

Proof. A standard procedure shows that

$$
\left\|\mathcal{I}_{n}^{*}\right\|_{\infty}=\max _{\mathbf{t} \in \Omega} \sum_{\mathbf{j} \in \mathbb{H}_{n}^{*}}\left|\Phi_{n}^{*}\left(\mathbf{t}-\frac{\mathbf{j}}{n}\right)\right| .
$$

By (3.9) and the fact that $D_{n}=\Theta_{n}-\Theta_{n-1}$, it is sufficient to show that

$$
\frac{1}{3 n^{2}} \max _{\mathbf{t} \in \Omega} \sum_{\mathbf{j} \in \mathbb{H}_{n}^{*}}\left|D_{n}\left(\mathbf{t}-\frac{\mathbf{j}}{n}\right)\right| \leq c(\log n)^{2}, \quad n \geq 0 .
$$

By (3.13) and (3.14), the estimate can be further reduced to show that

$$
\begin{aligned}
J_{n}(\mathbf{t}) & :=\frac{1}{3 n^{2}} \sum_{\mathbf{j} \in \mathbb{H}_{n}^{*}}\left|\frac{\sin \frac{n \pi}{3}\left(t_{2}-t_{3}-\frac{j_{2}-j_{3}}{n}\right)}{\sin \frac{\pi}{3}\left(t_{2}-t_{3}-\frac{j_{2}-j_{3}}{n}\right)} \frac{\sin \frac{(n+1) \pi}{3}\left(t_{1}-t_{3}-\frac{j_{1}-j_{3}}{n}\right)}{\sin \frac{\pi}{3}\left(t_{1}-t_{3}-\frac{j_{1}-j_{3}}{n}\right)}\right| \\
& :=\sum_{\mathbf{j} \in \mathbb{H}_{n}^{*}} F_{n}\left(\mathbf{t}-\frac{\mathbf{j}}{n}\right)
\end{aligned}
$$

and two other similar sums obtained by permuting $t_{1}, t_{2}, t_{3}$ are bounded by $c(\log n)^{2}$ for all $\mathbf{t} \in \Omega$. The three sums are similar, we only work with (3.16).

Fix a $\mathbf{t} \in \Omega$. If $s_{1}+s_{2}+s_{3}=0$, then the equations $s_{2}-s_{3}=t_{2}-t_{3}$ and $s_{1}-s_{3}=t_{1}-t_{3}$ has a unique solution $s_{2}=t_{2}$ and $s_{3}=t_{3}$. In particular, there can be at most one $\mathbf{j}$ such that the denominator of $F_{n}\left(\mathbf{t}-\frac{\mathbf{j}}{n}\right)$ is zero. For $\mathbf{t} \in \bar{\Omega}$, setting $s_{1}=\left(t_{1}-t_{3}\right) / 3=\left(2 t_{1}+t_{2}\right) / 3$ and $s_{2}=\left(t_{2}-t_{3}\right) / 3=\left(t_{1}+2 t_{2}\right) / 3$, it is easy to see that $-1 \leq s_{1}, s_{2} \leq 1$. The same consideration also shows that $-3 n \leq j_{1}-j_{3}, j_{2}-j_{3} \leq 3 n$ for $\mathbf{j} \in \mathbb{H}_{n}^{*}$. Consequently, enlarging the set over which the summation is taken, it follows that

$$
J_{n}(\mathbf{t}) \leq \frac{1}{3 n^{2}} \sum_{k_{2}=-3 n}^{3 n}\left|\frac{\sin n \pi\left(s_{2}-\frac{k_{2}}{3 n}\right)}{\sin \pi\left(s_{2}-\frac{k_{2}}{3 n}\right)}\right| \sum_{k_{1}=-3 n}^{3 n}\left|\frac{\sin (n+1) \pi\left(s_{1}-\frac{k_{1}}{3 n}\right)}{\sin \pi\left(s_{1}-\frac{k_{1}}{3 n}\right)}\right| .
$$

For $s \in[-1,1]$, a well-known procedure in one variable shows that

$$
\max _{s \in[-1,1]} \frac{1}{n} \sum_{k=-3 n}^{3 n}\left|\frac{\sin n \pi\left(s-\frac{k}{3 n}\right)}{\sin \pi\left(s-\frac{k}{3 n}\right)}\right| \leq c \log n,
$$

from which the stated results follows immediately. 


\section{Discrete Fourier analysis on the triangle}

Working with functions invariant under the isometries of the hexagon lattice, we can carry out a Fourier analysis on the equilateral triangle based on the analysis on the hexagon.

4.1. Generalized sine and cosine functions. The group $\mathcal{G}$ of isometries of the hexagon lattice is generated by the reflections in the edges of the equilateral triangles inside it, which is the reflection group $\mathcal{A}_{2}$. In homogeneous coordinates, the three reflections $\sigma_{1}, \sigma_{2}, \sigma_{3}$ are defined by

$$
\mathbf{t} \sigma_{1}:=-\left(t_{1}, t_{3}, t_{2}\right), \quad \mathbf{t} \sigma_{2}:=-\left(t_{2}, t_{1}, t_{3}\right), \quad \mathbf{t} \sigma_{3}:=-\left(t_{3}, t_{2}, t_{1}\right) .
$$

Because of the relations $\sigma_{3}=\sigma_{1} \sigma_{2} \sigma_{1}=\sigma_{2} \sigma_{1} \sigma_{2}$, the group $\mathcal{G}$ is given by

$$
\mathcal{G}=\left\{1, \sigma_{1}, \sigma_{2}, \sigma_{3}, \sigma_{1} \sigma_{2}, \sigma_{2} \sigma_{1}\right\}
$$

For a function $f$ in homogeneous coordinates, the action of the group $\mathcal{G}$ on $f$ is defined by $\sigma f(\mathbf{t})=f(\mathbf{t} \sigma), \sigma \in \mathcal{G}$. Following [9], we call a function $f$ invariant under $\mathcal{G}$ if $\sigma f=f$ for all $\sigma \in \mathcal{G}$, and call it anti-invariant under $\mathcal{G}$ if $\sigma f=\rho(\sigma) f$ for all $\sigma \in \mathcal{G}$, where $\rho(\sigma)=-1$ if $\sigma=\sigma_{1}, \sigma_{2}, \sigma_{3}$, and $\rho(\sigma)=1$ for other elements in $\mathcal{G}$. The following proposition is easy to verify $([\underline{9})$.

Proposition 4.1. Define two operators $\mathcal{P}^{+}$and $\mathcal{P}^{-}$acting on $f(\mathbf{t})$ by

$$
\mathcal{P}^{ \pm} f(\mathbf{t})=\frac{1}{6}\left[f(\mathbf{t})+f\left(\mathbf{t} \sigma_{1} \sigma_{2}\right)+f\left(\mathbf{t} \sigma_{2} \sigma_{1}\right) \pm f\left(\mathbf{t} \sigma_{1}\right) \pm f\left(\mathbf{t} \sigma_{2}\right) \pm f\left(\mathbf{t} \sigma_{3}\right)\right] .
$$

Then the operators $\mathcal{P}^{+}$and $\mathcal{P}^{-}$are projections from the class of $H$-periodic functions onto the class of invariant, respectively anti-invariant functions.

Recall that $\phi_{\mathbf{k}}(\mathbf{t})=\mathrm{e}^{\frac{2 \pi i}{3} \mathbf{k} \cdot \mathbf{t}}$. Following [21, we shall call the functions

$$
\begin{aligned}
\mathrm{TC}_{\mathbf{k}}(\mathbf{t}):=\mathcal{P}^{+} \phi_{\mathbf{k}}(\mathbf{t})= & \frac{1}{6}\left[\phi_{k_{1}, k_{2}, k_{3}}(\mathbf{t})+\phi_{k_{2}, k_{3}, k_{1}}(\mathbf{t})+\phi_{k_{3}, k_{1}, k_{2}}(\mathbf{t})\right. \\
& \left.+\phi_{-k_{1},-k_{3},-k_{2}}(\mathbf{t})+\phi_{-k_{2},-k_{1},-k_{3}}(\mathbf{t})+\phi_{-k_{3},-k_{2},-k_{1}}(\mathbf{t})\right], \\
\mathrm{TS}_{\mathbf{k}}(\mathbf{t}):=\frac{1}{i} \mathcal{P}^{-} \phi_{\mathbf{k}}(\mathbf{t})= & \frac{1}{6 i}\left[\phi_{k_{1}, k_{2}, k_{3}}(\mathbf{t})+\phi_{k_{2}, k_{3}, k_{1}}(\mathbf{t})+\phi_{k_{3}, k_{1}, k_{2}}(\mathbf{t})\right. \\
& \left.-\phi_{-k_{1},-k_{3},-k_{2}}(\mathbf{t})-\phi_{-k_{2},-k_{1},-k_{3}}(\mathbf{t})-\phi_{-k_{3},-k_{2},-k_{1}}(\mathbf{t})\right]
\end{aligned}
$$

a generalized cosine and a generalized sine, respectively, where the second equal sign follows from the fact that $\phi_{\mathbf{k}}(\mathbf{t} \sigma)=\phi_{\mathbf{k} \sigma}(\mathbf{t})$ for every $\sigma \in \mathcal{G}$. It follows that $\mathrm{TC}_{\mathbf{k}}$ and $\mathrm{TS}_{\mathbf{k}}$ are invariant and anti-invariant, respectively.

These two functions appear as eigenfunctions of the Laplacian on the triangle and they have been studied in [9, 12, 13, 15, 16, 21, 22, under various coordinate systems. In particular, they are shown to share many properties of the classical sine and cosine functions in homogeneous coordinates in [21, 22. Some of those properties that we shall need will be recalled below. The advantage of homogeneous coordinates lies in the symmetry of the formulas. For example, the equations $t_{1}+$ $t_{2}+t_{3}=0$ and $k_{1}+k_{2}+k_{3}=0$ can be used to write $\mathrm{TC}_{\mathbf{k}}$ and $\mathrm{TS}_{\mathbf{k}}$ in several useful 
forms, such as

$$
\begin{aligned}
\mathrm{TC}_{\mathbf{k}}(\mathbf{t})= & \frac{1}{3}\left[\mathrm{e}^{\frac{i \pi}{3}\left(k_{2}-k_{3}\right)\left(t_{2}-t_{3}\right)} \cos k_{1} \pi t_{1}\right. \\
& \left.+\mathrm{e}^{\frac{i \pi}{3}\left(k_{2}-k_{3}\right)\left(t_{3}-t_{1}\right)} \cos k_{1} \pi t_{2}+\mathrm{e}^{\frac{i \pi}{3}\left(k_{2}-k_{3}\right)\left(t_{1}-t_{2}\right)} \cos k_{1} \pi t_{3}\right], \\
\mathrm{TS}_{\mathbf{k}}(\mathbf{t})= & \frac{1}{3}\left[\mathrm{e}^{\frac{i \pi}{3}\left(k_{2}-k_{3}\right)\left(t_{2}-t_{3}\right)} \sin k_{1} \pi t_{1}\right. \\
& \left.+\mathrm{e}^{\frac{i \pi}{3}\left(k_{2}-k_{3}\right)\left(t_{3}-t_{1}\right)} \sin k_{1} \pi t_{2}+\mathrm{e}^{\frac{i \pi}{3}\left(k_{2}-k_{3}\right)\left(t_{1}-t_{2}\right)} \sin k_{1} \pi t_{3}\right],
\end{aligned}
$$

and similar formulas derived from the permutations of $t_{1}, t_{2}, t_{3}$. In particular, it follows from (4.3) that $\mathrm{TS}_{\mathbf{k}}(\mathbf{t}) \equiv 0$ whenever $\mathbf{k}$ contains one zero component.

For invariant functions, we can use symmetry to translate the results on the hexagon to one of its six equilateral triangles. We shall choose the triangle as

$$
\begin{aligned}
\Delta: & :=\left\{\left(t_{1}, t_{2}, t_{3}\right): t_{1}+t_{2}+t_{3}=0,0 \leq t_{1}, t_{2},-t_{3} \leq 1\right\} \\
& =\left\{\left(t_{1}, t_{2}\right): t_{1}, t_{2} \geq 0, t_{1}+t_{2} \leq 1\right\} .
\end{aligned}
$$

The region $\Delta$ and its relative position in the hexagon is depicted in Figure 5 .
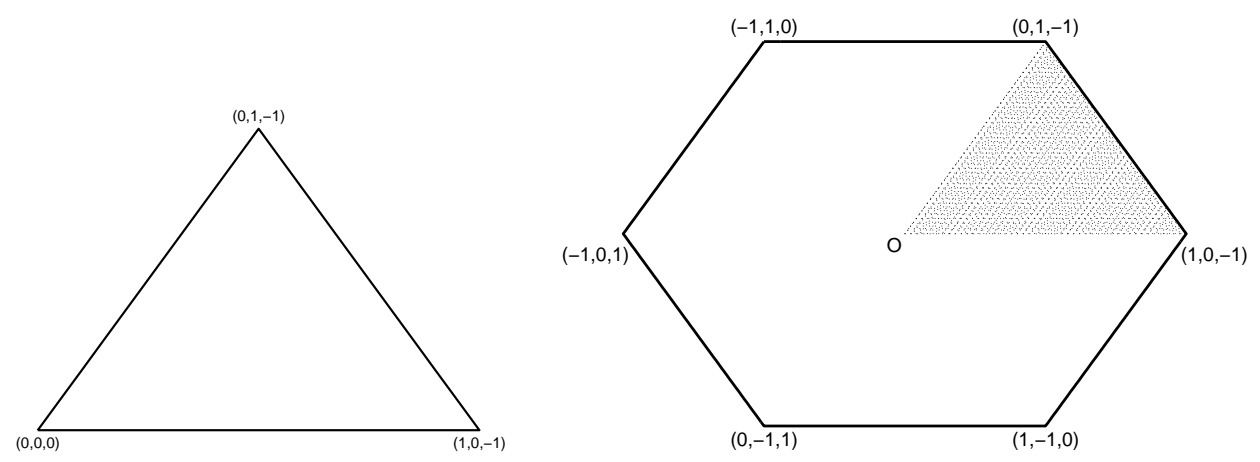

Figure 5. The equilateral triangle $\Delta$ in the hexagon.

The inner product on $\Delta$ is defined by

$$
\langle f, g\rangle_{\Delta}:=\frac{1}{|\Delta|} \int_{\Delta} f(\mathbf{t}) \overline{g(\mathbf{t})} d \mathbf{t}=2 \int_{\Delta} f\left(t_{1}, t_{2}\right) \overline{g\left(t_{1}, t_{2}\right)} d t_{1} d t_{2} .
$$

If $f \bar{g}$ is invariant under $\mathcal{G}$, then it is easy to see that $\langle f, g\rangle_{H}=\langle f, g\rangle_{\Delta}$.

When $\mathrm{TC}_{\mathbf{k}}$ are restricted to the triangle $\Delta$, we only need to consider a subset of $\mathbf{k} \in \mathbb{H}$. In fact, since $\mathrm{TC}_{\mathbf{k} \sigma}(\mathbf{t})=\mathrm{TC}_{\mathbf{k}}(\mathbf{t} \sigma)=\mathrm{TC}_{\mathbf{k}}(\mathbf{t})$ for $\mathbf{t} \in \Delta$ and $\sigma \in \mathcal{G}$, we can restrict $\mathbf{k}$ to the index set

$$
\Lambda:=\left\{\mathbf{k} \in \mathbb{H}: k_{1} \geq 0, k_{2} \geq 0, k_{3} \leq 0\right\} .
$$

We define the space $\mathcal{T}$ as the collection of finite linear combinations of elements in $\left\{\mathrm{TC}_{\mathbf{k}}, \mathrm{TS}_{\mathbf{k}}: \mathbf{k} \in \Lambda\right\}$; that is,

$$
\mathcal{T}=\left\{\sum_{\mathbf{k} \in \Xi}\left(a_{\mathbf{k}} \mathrm{TC}_{\mathbf{k}}+b_{\mathbf{k}} \mathrm{TS}_{\mathbf{k}}\right): a_{\mathbf{k}}, b_{\mathbf{k}} \in \mathbb{C}, \Xi \subset \Lambda, \# \Xi<\infty\right\} .
$$


Since $\mathrm{TS}_{\mathbf{k}}(\mathbf{t})=0$ whenever $\mathbf{k}$ has a zero component, $\mathrm{TS}_{\mathbf{k}}(\mathbf{t})$ are defined only for $\mathbf{k} \in \Lambda^{\circ}$, where

$$
\Lambda^{\circ}:=\left\{\mathbf{k} \in \mathbb{H}: k_{1}>0, k_{2}>0, k_{3}<0\right\}
$$

which is the set of the interior points of $\Lambda$. These functions are orthogonal on $\Delta$.

Proposition 4.2. For $\mathbf{k}, \mathbf{j} \in \Lambda$,

$$
\left\langle\mathrm{TC}_{\mathbf{k}}, \mathrm{TC}_{\mathbf{j}}\right\rangle_{\Delta}=\delta_{\mathbf{k}, \mathbf{j}} \begin{cases}1, & \mathbf{k}=0 \\ \frac{1}{3}, & \mathbf{k} \in \Lambda \backslash \Lambda^{\circ}, \mathbf{k} \neq 0, \\ \frac{1}{6}, & \mathbf{k} \in \Lambda^{\circ},\end{cases}
$$

and for $\mathbf{k}, \mathbf{j} \in \Lambda^{\circ}$,

$$
\left\langle\mathrm{TS}_{\mathbf{k}}, \mathrm{TS}_{\mathbf{j}}\right\rangle_{\Delta}=\frac{1}{6} \delta_{\mathbf{k}, \mathbf{j}}
$$

This follows easily from $\langle f, g\rangle_{H}=\langle f, g\rangle_{\Delta}$, which holds if $f \bar{g}$ is invariant, the fact that $T C_{\mathbf{k}}$ is invariant and $T S_{\mathbf{k}}$ is anti-invariant, and the orthogonality of $\phi_{\mathbf{k}}$ in (3.3). The norms of $\mathrm{TC}_{\mathbf{k}}$ are divided into three cases, since $\mathrm{TC}_{0}(\mathbf{t})=1$ and

$$
\mathrm{TC}_{\mathbf{k}}(\mathbf{t})=\frac{1}{3}\left[\phi_{k_{1}, k_{2}, k_{3}}(\mathbf{t})+\phi_{k_{2}, k_{3}, k_{1}}(\mathbf{t})+\phi_{k_{3}, k_{1}, k_{2}}(\mathbf{t})\right], \quad k_{1} k_{2} k_{3}=0 .
$$

4.2. Discrete inner product on the triangle. Using the fact that $T C_{k}$ and $T S_{k}$ are invariant and anti-invariant under $\mathcal{G}$ and the orthogonality of $\phi_{\mathbf{k}}$ with respect to the symmetric inner product $\langle\cdot, \cdot\rangle_{n}^{*}$, we can deduce a discrete orthogonality for the generalized cosine and sine functions. For this purpose, we define

$$
\Lambda_{n}:=\left\{\mathbf{k} \in \Lambda:-k_{3} \leq n\right\}=\left\{\left(k_{1}, k_{2}\right) \in \mathbb{Z}^{2}: k_{1} \geq 0, k_{2} \geq 0, k_{1}+k_{2} \leq n\right\},
$$

and denote by $\Lambda^{\circ}, \Lambda_{n}^{\mathrm{e}}, \Lambda_{n}^{\mathrm{v}}$ the set of points in the interior of $\Lambda_{n}$, on the boundary of $\Lambda_{n}$ but not vertices, and at vertices, respectively; that is,

$$
\begin{aligned}
& \Lambda_{n}^{\circ}:=\left\{\mathbf{k} \in \Lambda_{n}: k_{1}>0, k_{2}>0,-k_{3}<n\right\}, \\
& \Lambda_{n}^{\mathrm{v}}:=\{(n, 0,-n),(0, n,-n),(0,0,0)\}, \\
& \Lambda_{n}^{\mathrm{e}}:=\left\{\mathbf{k} \in \Lambda_{n}: \mathbf{k} \notin \Lambda_{n}^{\circ} \cup \Lambda^{\mathrm{v}}\right\} .
\end{aligned}
$$

We then define by $\mathcal{T} C_{n}$ and $\mathcal{T} S_{n}$ the subspace of $\mathcal{T}$ defined by

$$
\mathcal{T} C_{n}=\operatorname{span}\left\{\mathrm{TC}_{\mathbf{k}}: \mathbf{k} \in \Lambda_{n}\right\} \quad \text { and } \mathcal{T} S_{n}=\operatorname{span}\left\{\mathrm{TS}_{\mathbf{k}}: \mathbf{k} \in \Lambda_{n}^{\circ}\right\},
$$

respectively.

Theorem 4.3. Let the discrete inner product $\langle\cdot, \cdot\rangle_{\Delta, n}$ be defined by

$$
\langle f, g\rangle_{\Delta, n}=\frac{1}{3 n^{2}} \sum_{\mathbf{j} \in \Lambda_{n}} \lambda_{\mathbf{j}}^{(n)} f\left(\frac{\mathbf{j}}{n}\right) \overline{g\left(\frac{\mathbf{j}}{n}\right)},
$$

where

$$
\lambda_{\mathbf{j}}^{(n)}:= \begin{cases}6, & \mathbf{j} \in \Lambda^{\circ}, \\ 3, & \mathbf{j} \in \Lambda^{\mathrm{e}}, \\ 1, & \mathbf{j} \in \Lambda^{\mathrm{v}} .\end{cases}
$$

Then

$$
\langle f, g\rangle_{\Delta}=\langle f, g\rangle_{\Delta, n}, \quad f, g \in \mathcal{T} C_{n} .
$$


Proof. We shall deduce the result from the discrete inner product $\langle\cdot, \cdot\rangle_{n}^{*}$ defined in (3.6). Let $\mathbf{j} \mathcal{G}$ denote the orbit of $\mathbf{j}$ under $\mathcal{G}$, which is the set $\{\mathbf{j} \sigma: \sigma \in \mathcal{G}\}$. It is easy to see that for $\mathbf{j} \in \mathbb{H}$,

$$
|\mathbf{j} \mathcal{G}|=\left\{\begin{array}{ll}
6 & \text { if } j_{1} j_{2} j_{3} \neq 0 \\
3 & \text { if } j_{1} j_{2} j_{3}=0 \\
1 & \text { if } \mathbf{j}=(0,0,0)
\end{array} \text { but } \mathbf{j} \neq(0,0,0) .\right.
$$

Assume that $f$ is invariant. Using (4.11), we see that

$$
\begin{aligned}
\sum_{\mathbf{j} \in \mathbb{H}_{n}^{*}, j_{1} j_{2} j_{3} \neq 0} c_{\mathbf{j}}^{(n)} f\left(\frac{\mathbf{j}}{n}\right) & =6 \sum_{\mathbf{j} \in \Lambda_{n}^{\circ}} c_{\mathbf{j}}^{(n)} f\left(\frac{\mathbf{j}}{n}\right)+6 \sum_{\mathbf{j} \in \Lambda_{n}^{\mathrm{e}}, j_{1} j_{2} j_{3} \neq 0} c_{\mathbf{j}}^{(n)} f\left(\frac{\mathbf{j}}{n}\right) \\
& =\sum_{\mathbf{j} \in \Lambda_{n}, j_{1} j_{2} j_{3} \neq 0} \lambda_{\mathbf{j}}^{(n)} f\left(\frac{\mathbf{j}}{n}\right),
\end{aligned}
$$

and

$$
\begin{aligned}
\sum_{\mathbf{j} \in \mathbb{H}_{n}^{*}, j_{1} j_{2} j_{3}=0} c_{\mathbf{j}}^{(n)} f\left(\frac{\mathbf{j}}{n}\right) & =f(0,0,0)+3 \sum_{j \in \Lambda^{\mathrm{e}}, j_{1} j_{2} j_{3}=0} c_{\mathbf{j}}^{(n)} f\left(\frac{\mathbf{j}}{n}\right)+3 \sum_{j \in \Lambda^{v}, \mathbf{j} \neq 0} c_{\mathbf{j}}^{(n)} f\left(\frac{\mathbf{j}}{n}\right) \\
& =\sum_{\mathbf{j} \in \Lambda_{n}, j_{1} j_{2} j_{3}=0} \lambda_{\mathbf{j}}^{(n)} f\left(\frac{\mathbf{j}}{n}\right) .
\end{aligned}
$$

Replacing $f$ by $f \bar{g}$ and adding these two sums, we have proved that $\langle f, g\rangle_{n}^{*}=$ $\langle f, g\rangle_{\Delta, n}$ for invariant $f \bar{g}$, which applies to $f, g \in \mathcal{T} C_{n-1}$. Thus, the stated result follows immediately from Theorem 3.3 and the fact that $\langle f, g\rangle_{H}=\langle f, g\rangle_{\Delta}$.

The proof of the above proposition also applies to $f, g \in \mathcal{T} S_{n}$ as $f \bar{g}$ is invariant if both $f$ and $g$ are anti-invariant. Noticing also that $\mathcal{T} S_{\mathbf{j}}\left(\frac{\mathbf{j}}{n}\right)=0$ when $\mathbf{j} \in \Lambda^{\mathrm{e}}$, and hence we conclude the following result.

Proposition 4.4. Let the discrete inner product $\langle\cdot, \cdot\rangle_{\Delta^{\circ}, n}$ be defined by

$$
\langle f, g\rangle_{\Delta^{\circ}, n}=\frac{2}{n^{2}} \sum_{\mathbf{j} \in \Lambda_{n}^{\circ}} f\left(\frac{\mathbf{j}}{n}\right) \overline{g\left(\frac{\mathbf{j}}{n}\right)} .
$$

Then

$$
\langle f, g\rangle_{\Delta}=\langle f, g\rangle_{\Delta^{\circ}, n}, \quad f, g \in \mathcal{T} S_{n} .
$$

The relation (4.10) can be regarded as a cubature formula

$$
\frac{1}{|\Delta|} \int_{\Delta} f(\mathbf{t}) d \mathbf{t}=\frac{1}{3 n^{2}} \sum_{\mathbf{j} \in \Lambda_{n}} \lambda_{\mathbf{j}}^{(n)} f\left(\frac{\mathbf{j}}{n}\right)
$$

which is exact for $f=g \bar{h}, g, h \in \mathcal{T} C_{n}$. It turns out, however, that much more is true. We state the following result in terms of the triangle in $\left(t_{1}, t_{2}\right)$.

Theorem 4.5. For $n \geq 0$, the cubature formula

$$
2 \int_{\Delta} f\left(t_{1}, t_{2}\right) d t_{1} d t_{2}=\frac{1}{3 n^{2}} \sum_{j_{1}=0}^{n} \sum_{j_{2}=0}^{j_{1}} \lambda_{\mathbf{j}}^{(n)} f\left(\frac{j_{1}}{n}, \frac{j_{2}}{n}\right)
$$

is exact for all $f \in \mathcal{T} C_{2 n-1}$. 


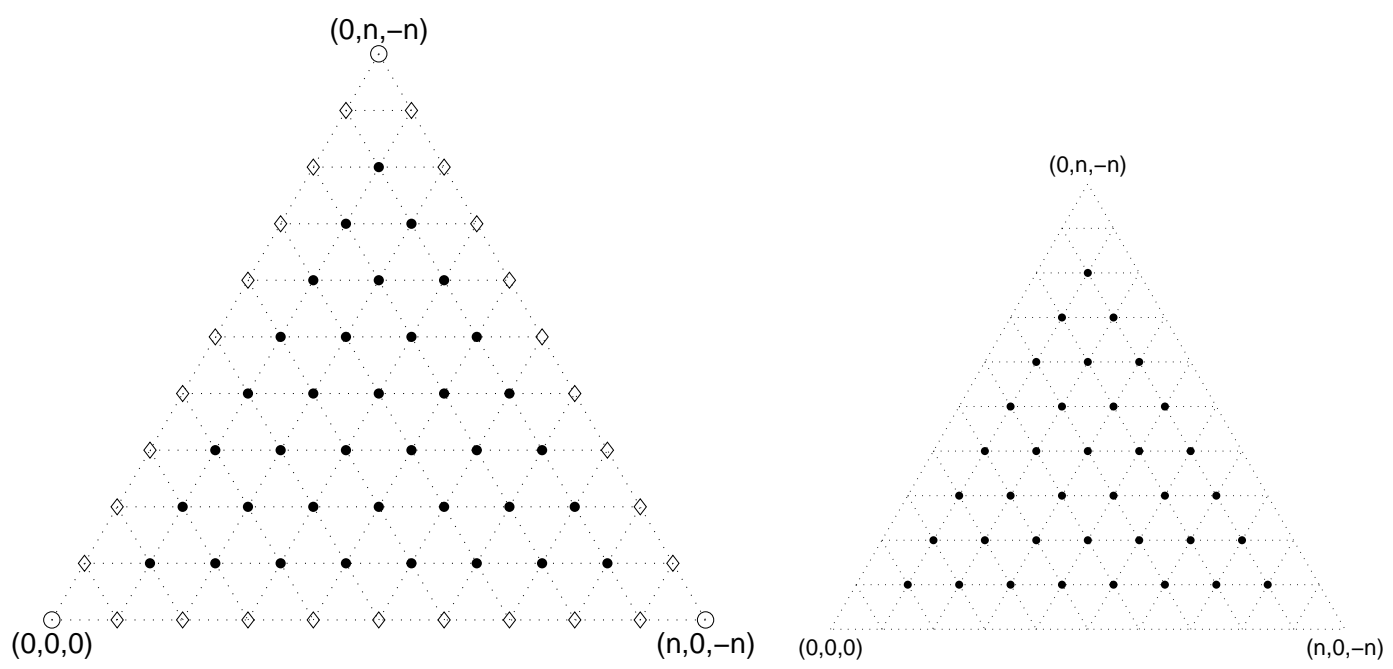

Figure 6. Left: $\Lambda_{n}$ in which $\bullet, \diamond$ and $\circ$ correspond to points of different coefficients in $\langle\cdot, \cdot\rangle_{n}^{*}$. Right: • indicates points in $\Lambda^{\circ}$.

In order to establish such a result, we will need to study the structure of $\mathcal{T} C_{n}$, which, and the proof of the theorem, will be given in Section 5. One may note that the formulation of the result resembles a Gaussian quadrature. There is indeed a connection which will also be explored in Section 5 .

4.3. Interpolation on the triangle. One way to deduce the result on interpolation is by making use of the orthogonality of generalized trigonometric functions with respect to the discrete inner product, as shown in the first theorem below. Recall the operator $\mathcal{P}^{ \pm}$defined in (4.1).

Theorem 4.6. For $n \geq 0$ and $f \in C(\Delta)$ define

$$
\mathcal{L}_{n} f(\mathbf{t}):=\sum_{\mathbf{j} \in \Lambda_{n}^{\circ}} f\left(\frac{\mathbf{j}}{n}\right) \ell_{\mathbf{j}, n}^{\circ}(\mathbf{t}), \quad \ell_{\mathbf{j}, n}^{\circ}(\mathbf{t})=\frac{2}{n^{2}} \sum_{\mathbf{k} \in \Lambda_{n}^{\circ}} \mathrm{TS}_{\mathbf{k}}(\mathbf{t}) \overline{\mathrm{TS}_{\mathbf{k}}\left(\frac{\mathbf{j}}{n}\right)} .
$$

Then $\mathcal{L}_{n}$ is the unique function in $\mathcal{T} S_{n}$ that satisfies

$$
\mathcal{L}_{n} f\left(\frac{\mathbf{j}}{n}\right)=f\left(\frac{\mathbf{j}}{n}\right), \quad \mathbf{j} \in \Lambda_{n}^{\circ} .
$$

Furthermore, the fundamental interpolation function $\ell_{\mathbf{j}, n}^{\circ}$ is real and satisfies

$$
\ell_{\mathbf{j}, n}^{\circ}(\mathbf{t})=\frac{1}{3 n^{2}} \mathcal{P}_{\mathbf{t}}^{-}\left[\Theta_{n-1}\left(\mathbf{t}-\frac{\mathbf{j}}{n}\right)-\Theta_{n-2}\left(\mathbf{t}-\frac{\mathbf{j}}{n}\right)\right],
$$

where $\mathcal{P}_{\mathbf{t}}^{-}$means that the operator $\mathcal{P}^{-}$is acting on the the variable $\mathbf{t}$ and $\Theta_{n}$ is defined in (3.10).

Proof. By (4.8) and (4.12), $\left\langle\mathrm{TS}_{\mathbf{j}}, \mathrm{TS}_{\mathbf{k}}\right\rangle_{\Delta^{\circ}, n}=\frac{1}{6} \delta_{\mathbf{k}, \mathbf{j}}$, which shows that $\ell_{\mathbf{j}, n}^{\circ}\left(\frac{\mathbf{k}}{n}\right)=\delta_{\mathbf{k}, \mathbf{j}}$ and verifies the interpolation condition. Furthermore, since $\operatorname{TS}_{\mathbf{k}}\left(\frac{\mathbf{j}}{n}\right)=\mathrm{TS}_{\mathbf{j}}\left(\frac{\mathbf{k}}{n}\right)=0$ whenever $\mathbf{k} \in \Lambda_{n} \backslash \Lambda_{n}^{\circ}$, and $\mathrm{TS}_{\mathbf{j}}(\mathbf{t})=0$ whenever $j_{1} j_{2} j_{3}=0$, it follows from the definition of $\mathrm{TS}_{\mathbf{j}}$ that

$$
\ell_{\mathbf{j}, n}^{\circ}(\mathbf{t})=\frac{1}{3 n^{2}} \mathcal{P}_{\mathbf{t}}^{-} \mathcal{P}_{\mathbf{j}}^{-} \sum_{\mathbf{k} \in \mathbb{H}_{n}^{\circ}} \phi_{\mathbf{k}}(\mathbf{t}) \overline{\phi_{\mathbf{k}}\left(\frac{\mathbf{j}}{n}\right)}=\frac{1}{3 n^{2}} \mathcal{P}_{\mathbf{t}}^{-} \mathcal{P}_{\mathbf{j}}^{-} D_{n-1}\left(\mathbf{t}-\frac{\mathbf{j}}{n}\right),
$$


where $D_{n}$ is defined in (3.12). To complete the proof we use the fact that if $F$ is an invariant function, then

$$
\mathcal{P}_{\mathbf{t}}^{-} \mathcal{P}_{\mathbf{s}}^{-} F(\mathbf{t}-\mathbf{s})=\mathcal{P}_{\mathbf{t}}^{-} F(\mathbf{t}-s),
$$

which can be easily verified by using the fact that the elements of the group $\mathcal{G}$ satisfy $\sigma_{i}^{2}=1, i=1,2,3$ and $\sigma_{3}=\sigma_{1} \sigma_{2} \sigma_{1}=\sigma_{2} \sigma_{1} \sigma_{2}$.

We can derive the result of interpolation on the $\Lambda_{n}$ using the same approach, but it is more illustrative to derive it from the interpolation on the hexagon given in Theorem 3.5.

Theorem 4.7. For $n \geq 0$ and $f \in C(\Delta)$ define

$$
\mathcal{L}_{n}^{*} f(\mathbf{t}):=\sum_{\mathbf{j} \in \Lambda_{n}} f\left(\frac{\mathbf{j}}{n}\right) \ell_{\mathbf{j}, n}^{\Delta}(\mathbf{t}), \quad \ell_{\mathbf{j}, n}^{\Delta}(\mathbf{t})=\lambda_{\mathbf{j}}^{(n)} \mathcal{P}^{+} \ell_{\mathbf{j}, n}(\mathbf{t}),
$$

where $\lambda_{\mathbf{j}}^{(n)}$ and $\ell_{\mathbf{j}, n}$ are defined in (4.9) and (3.7), respectively. Then $\mathcal{L}_{n}^{*}$ is the unique function in $\mathcal{T} C_{n}$ that satisfies

$$
\mathcal{L}_{n}^{*} f\left(\frac{\mathbf{j}}{n}\right)=f\left(\frac{\mathbf{j}}{n}\right), \quad \mathbf{j} \in \Lambda_{n} .
$$

Furthermore, the fundamental interpolation function $\ell_{\mathbf{j}, n}$ is given by

$\ell_{\mathbf{j}, n}^{\Delta}(\mathbf{t})=\frac{\lambda_{\mathbf{j}}^{(n)}}{3 n^{2}}\left[\frac{1}{2} \mathcal{P}^{+}\left(\Theta_{n}\left(\mathbf{t}-\frac{\mathbf{j}}{n}\right)-\Theta_{n-2}\left(\mathbf{t}-\frac{\mathbf{j}}{n}\right)\right)-\Re\left\{\mathrm{TC}_{n, 0,-n}(\mathbf{t}) \overline{\mathrm{TC}_{n, 0,-n}\left(\frac{\mathbf{j}}{n}\right)}\right\}\right]$.

Proof. Throughout this proof, write $\ell_{\mathbf{j}}=\ell_{\mathbf{j}, n}$. Recall the equation (3.11), which states that $\ell_{\mathbf{j}}\left(\frac{\mathbf{k}}{n}\right)=1$ if $\mathbf{k} \equiv \mathbf{j} \bmod 3$ and $\ell_{\mathbf{j}}\left(\frac{\mathbf{k}}{n}\right)=0$ otherwise. Let $\mathbf{k} \in \Lambda$. If $\mathbf{j} \in \Lambda^{\circ}$, then we have $\mathcal{P}^{+} \ell_{\mathbf{j}}\left(\frac{\mathbf{k}}{n}\right)=\frac{1}{6} \ell_{\mathbf{j}}\left(\frac{\mathbf{k}}{n}\right)=\left[\lambda_{\mathbf{j}}^{(n)}\right]^{-1} \delta_{\mathbf{k}, \mathbf{j}}$. If $\mathbf{j} \in \Lambda^{\mathrm{e}}$ and $j_{1} j_{2} j_{3} \neq 0$, then $\mathcal{P}^{+} \ell_{\mathbf{j}}\left(\frac{\mathbf{k}}{n}\right)=\frac{1}{6}\left[\ell_{\mathbf{j}}\left(\frac{\mathbf{k}}{n}\right)+\ell_{\mathbf{j}}\left(\frac{\mathbf{k}^{*}}{n}\right)\right]=\frac{1}{3} \delta_{\mathbf{k}, \mathbf{j}}=\left[\lambda_{\mathbf{j}}^{(n)}\right]^{-1} \delta_{\mathbf{k}, \mathbf{j}}$, where $k^{*}$ is defined as in the proof of Theorem 3.5. If $\mathbf{j} \in \Lambda^{\mathrm{e}}$ and $j_{1} j_{2} j_{3}=0$, then $\mathcal{P}^{+} \ell_{\mathbf{j}}\left(\frac{\mathbf{k}}{n}\right)=$ $\frac{1}{3} \ell_{\mathbf{j}}\left(\frac{\mathbf{k}}{n}\right)=\left[\lambda_{\mathbf{j}}^{(n)}\right]^{-1} \delta_{\mathbf{k}, \mathbf{j}}$ since $\mathbf{j} \in \mathbb{H}_{n}^{\circ}$. If $\mathbf{j} \in \Lambda^{\mathrm{v}}$ and $\mathbf{j} \neq(0,0,0)$, then $\mathcal{P}^{+} \ell_{\mathbf{j}}\left(\frac{\mathbf{k}}{n}\right)=$ $\frac{1}{3} \sum_{\mathbf{l} \in \mathcal{S}_{\mathbf{j}}} \ell_{\mathbf{l}}\left(\frac{\mathbf{k}}{n}\right)=\delta_{\mathbf{j}, \mathbf{k}}=\left[\lambda_{\mathbf{j}}^{(n)}\right]^{-1} \delta_{\mathbf{k}, \mathbf{j}}$, where $\mathcal{S}_{\mathbf{j}}$ is defined as in the proof of Theorem 3.5. Finally, if $\mathbf{j}=(0,0,0), \mathcal{P}^{+} \ell_{\mathbf{j}}\left(\frac{\mathbf{k}}{n}\right)=\delta_{\mathbf{k}, \mathbf{j}}=\left[\lambda_{\mathbf{j}}^{(n)}\right]^{-1} \delta_{\mathbf{k}, \mathbf{j}}$. Putting these together, we have proved that $\ell_{\mathbf{j}}^{\Delta}\left(\frac{\mathbf{k}}{n}\right)=\delta_{\mathbf{k}, \mathbf{j}}$, which verifies the interpolation condition.

The explicit formula of $\ell_{\mathbf{j}}^{\Delta}$ follows from (3.9) and the fact that

$$
\begin{aligned}
\mathcal{P}^{+} \sum_{\mathbf{k} \in \mathbb{H}_{n}^{v}} \phi_{\mathbf{k}}\left(\mathbf{t}-\frac{\mathbf{j}}{n}\right) & =\mathcal{P}^{+} \sum_{\mathbf{k} \in \mathbb{H}_{n}^{v}} \phi_{\mathbf{k}}(\mathbf{t}) \overline{\phi_{\mathbf{k}}\left(\frac{\mathbf{j}}{n}\right)}=\sum_{\mathbf{k} \in \mathbb{H}_{n}^{\mathbf{v}}} \mathrm{TC}_{\mathbf{k}}(\mathbf{t}) \overline{\phi_{\mathbf{k}}\left(\frac{\mathbf{j}}{n}\right)} \\
& =3 \mathrm{TC}_{n, 0,-n}(\mathbf{t}) \overline{\mathrm{TC}_{n, 0,-n}\left(\frac{\mathbf{j}}{n}\right)}+3 \mathrm{TC}_{0, n,-n}(\mathbf{t}) \overline{\mathrm{TC}_{0, n,-n}\left(\frac{\mathbf{j}}{n}\right)},
\end{aligned}
$$

as well as the fact that $\overline{\mathrm{TC}_{0, n,-n}(\mathbf{t})}=\mathrm{TC}_{n, 0,-n}(\mathbf{t})$.

As a consequence of Theorem 3.6, we immediately have the following:

Theorem 4.8. Let $\left\|\mathcal{L}_{n}\right\|$ and $\left\|\mathcal{L}_{n}^{*}\right\|$ denote the operator norm of $\mathcal{L}_{n}: C(\Delta) \mapsto C(\Delta)$ and $\mathcal{L}_{n}^{*}: C(\Delta) \mapsto C(\Delta)$, respectively. Then there is a constant $c$, independent of $n$, such that

$$
\left\|\mathcal{L}_{n}\right\| \leq c(\log n)^{2} \quad \text { and } \quad\left\|\mathcal{L}_{n}^{*}\right\| \leq c(\log n)^{2} .
$$

In particular, if $f \in C^{1}(\Omega)$, the class of functions having continuous derivatives on $\Omega$, then both $\mathcal{L}_{n} f$ and $\mathcal{L}_{n}^{*} f$ converge uniformly to $f$ on $\Omega$. 
This theorem shows a sharp contrast between interpolation by trigonometric functions on $\Lambda_{n}$ or $\Lambda_{n}^{\circ}$ and the interpolation by algebraic polynomials on $\Lambda_{n}$. From the result in one variable [17, the Lebesgue constant for algebraic polynomial interpolation grows exponentially as $n \rightarrow \infty$ and, as a consequence, the convergence does not hold in $C^{1}(\Omega)$.

\section{Generalized Chebyshev polynomials and their zeros}

Just like in the classical case of one variable, we can use the generalized sine and cosine functions to define analogues of Chebyshev polynomials of the first and the second kind, respectively, which are orthogonal polynomials of the two variables.

5.1. Generalized Chebyshev polynomials. To see the polynomial structure among the generalized trigonometric functions, we make a change of variables. Denote

$$
z:=\mathrm{TC}_{0,1,-1}(\mathbf{t})=\frac{1}{3}\left[\phi_{0,1,-1}(\mathbf{t})+\phi_{1,-1,0}(\mathbf{t})+\phi_{-1,0,1}(\mathbf{t})\right],
$$

whose real part and imaginary part, after simplification using (3.15), are

$$
\begin{aligned}
& x=\Re z=\frac{4}{3} \cos \frac{\pi}{3}\left(t_{2}-t_{1}\right) \cos \frac{\pi}{3}\left(t_{3}-t_{2}\right) \cos \frac{\pi}{3}\left(t_{1}-t_{3}\right)-\frac{1}{3}, \\
& y=\Im z=\frac{4}{3} \sin \frac{\pi}{3}\left(t_{2}-t_{1}\right) \sin \frac{\pi}{3}\left(t_{3}-t_{2}\right) \sin \frac{\pi}{3}\left(t_{1}-t_{3}\right),
\end{aligned}
$$

respectively. If we change variables $\left(t_{1}, t_{2}\right) \mapsto(x, y)$, then the region $\Delta$ is mapped onto the region $\Delta^{*}$ bounded by Steiner's hypocycloid,

$$
\Delta^{*}=\left\{(x, y):-3\left(x^{2}+y^{2}+1\right)^{2}+8\left(x^{3}-3 x y^{2}\right)+4 \geq 0\right\} \text {. }
$$

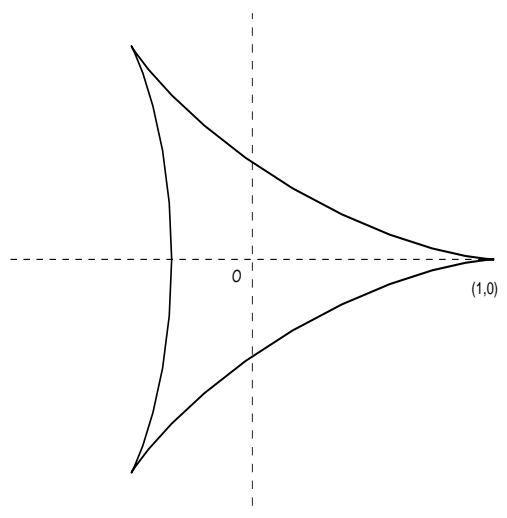

FiguRE 7 . The region $\triangle^{*}$ bounded by Steiner's hypocycloid.

Definition 5.1. Under the change of variables (5.1), define

$$
\begin{aligned}
& T_{k}^{m}(z, \bar{z}):=\mathrm{TC}_{k, m-k,-m}(\mathbf{t}), \quad 0 \leq k \leq m, \\
& U_{k}^{m}(z, \bar{z}):=\frac{\mathrm{TS}_{k+1, m-k+1,-m-2}(\mathbf{t})}{\mathrm{TS}_{1,1,-2}(\mathbf{t})}, \quad 0 \leq k \leq m .
\end{aligned}
$$

We will also use the notation $T_{k}^{m}(\mathbf{t})$ and $U_{k}^{m}(\mathbf{t})$ when it is more convenient to work with $\mathbf{t}$ variable. 
These functions are in fact polynomials of degree $m$ in the $z$ and $\bar{z}$ variables since they both satisfy a simple recursive relation.

Proposition 5.2. Let $P_{k}^{m}$ denote either $T_{k}^{m}$ or $U_{k}^{m}$. Then

$$
P_{m-k}^{m}(z, \bar{z})=\overline{P_{k}^{m}(z, \bar{z})}, \quad 0 \leq k \leq m,
$$

and they satisfy the recursion relation

$$
P_{k}^{m+1}(z, \bar{z})=3 z P_{k}^{m}(z, \bar{z})-P_{k+1}^{m}(z, \bar{z})-P_{k-1}^{m-1}(z, \bar{z})
$$

for $0 \leq k \leq m$ and $m \geq 1$, where we use

$$
\begin{aligned}
& T_{-1}^{m}(z, \bar{z})=T_{1}^{m+1}(z, \bar{z}), \quad T_{m+1}^{m}(z, \bar{z})=T_{m}^{m+1}(z, \bar{z}), \\
& U_{-1}^{m}(z, \bar{z})=0, \quad U_{m}^{m-1}(z, \bar{z})=0 .
\end{aligned}
$$

In particular, we have

$$
\begin{array}{lll}
T_{0}^{0}(z, \bar{z})=1, & T_{0}^{1}(z, \bar{z})=z, & T_{1}^{1}(z, \bar{z})=\bar{z}, \\
U_{0}^{0}(z, \bar{z})=1, & U_{0}^{1}(z, \bar{z})=3 z, & U_{1}^{1}(z, \bar{z})=3 \bar{z} .
\end{array}
$$

The recursion relation (5.5) follows from a straightforward computation. Together, (5.5) and (5.4) determine all $P_{k}^{m}$ recursively, which show that both $T_{k}^{m}$ and $U_{k}^{m}$ are polynomials of degree $m$ in $z$ and $\bar{z}$. Furthermore, each family inherits an orthogonal relation from the generalized trigonometric functions, so that they are orthogonal polynomials of two variables. They were studied by Koornwinder ([9, 10]), who called them the generalized Chebyshev polynomials of the first and the second kind, respectively. Moreover, they are special cases of orthogonal polynomials with respect to the weighted inner product

$$
\langle f, g\rangle_{w_{\alpha}}=c_{\alpha} \int_{\Delta^{*}} f(x, y) \overline{g(x, y)} w_{\alpha}(x, y) d x d y
$$

where $w_{\alpha}$ is the weight function defined in terms of the Jacobian of the change of variables (5.1),

$$
\begin{aligned}
w_{\alpha}(x, y)=\left|\frac{\partial(x, y)}{\partial\left(t_{1}, t_{2}\right)}\right|^{2 \alpha} & =\left[\frac{16}{27} \pi^{2} \sin \pi t_{1} \sin \pi t_{2} \sin \pi\left(t_{1}+t_{2}\right)\right]^{2 \alpha} \\
& =\frac{4^{\alpha}}{27^{\alpha}} \pi^{2 \alpha}\left[-3\left(x^{2}+y^{2}+1\right)^{2}+8\left(x^{3}-3 x y^{2}\right)+4\right]^{\alpha}
\end{aligned}
$$

and $c_{\alpha}$ is a normalization constant, $c_{\alpha}:=1 / \int_{\Delta} w_{\alpha}(x, y) d x d y$. We have, in particular, that $c_{-\frac{1}{2}}=2$ and $c_{\frac{1}{2}}=81 /\left(8 \pi^{4}\right)$. Since the change of variables (5.1) shows immediately that

$$
\frac{1}{|\Delta|} \int_{\Delta} f(\mathbf{t}) d \mathbf{t}=c_{-\frac{1}{2}} \int_{\Delta^{*}} f(x, y) w_{-\frac{1}{2}}(x, y) d x d y
$$

it follows from the orthogonality of $\mathrm{TC}_{\mathbf{j}}$ and $\mathrm{TS}_{\mathbf{j}}$ that $T_{k}^{m}$ and $U_{k}^{m}$ are orthogonal polynomials with respect to $w_{-\frac{1}{2}}$ and $w_{\frac{1}{2}}$, respectively. Furthermore, from Proposition 4.2 we have

$$
\left\langle T_{k}^{m}, T_{j}^{m}\right\rangle_{w_{-\frac{1}{2}}}=d_{k}^{m} \delta_{k, j}, \quad d_{k}^{m}:= \begin{cases}1, & m=k=0, \\ \frac{1}{3}, & k=0 \text { or } k=m, m>0, \\ \frac{1}{6}, & 1 \leq k \leq m-1, m>0\end{cases}
$$


and

$$
\left\langle U_{k}^{m}, U_{j}^{m}\right\rangle_{w_{\frac{1}{2}}}=\frac{1}{6} \delta_{k, j}, \quad 0 \leq j, k \leq m .
$$

Let $\widetilde{T}_{k}^{m}=\sqrt{d_{k}^{m}} T_{k}^{m}$ and $\widetilde{U}_{k}^{m}=\sqrt{6} U_{k}^{m}$. These are orthonormal polynomials. We introduce the notation

$$
\mathbb{P}_{m}=\left\{P_{0}^{m}, P_{1}^{m}, \ldots, P_{m}^{m}\right\}
$$

for either $P_{k}^{m}=\widetilde{T}_{k}^{m}$ or $\widetilde{U}_{k}^{m}$. The polynomials in $\mathbb{P}_{n}$ consist of an orthonormal basis for the space $\mathcal{V}_{n}$ of orthogonal polynomials of degree $m$. Evidently both $T_{k}^{m}$ and $U_{k}^{m}$ are complex polynomials. As a consequence of (5.4), however, it is easy to derive real orthogonal polynomials with respect to $w_{ \pm \frac{1}{2}}(x, y)$ on $\Delta^{*}$. In fact, let $\mathbb{P}_{m}$ be as in (5.8). Then the polynomials in the set $\mathbb{P}_{m}$ defined by

$$
\begin{aligned}
\mathbb{P}_{2 m-1}^{R}(x, y) & :=\left\{\sqrt{2} \Re\left\{P_{k}^{2 m-1}(z, \bar{z})\right\}, \sqrt{2} \Im\left\{P_{k}^{2 m-1}(z, \bar{z})\right\}, 0 \leq k \leq\lfloor m-1\rfloor\right\} \\
\mathbb{P}_{2 m}^{R}(x, y) & :=\left\{P_{m}^{2 m}, \sqrt{2} \Re\left\{P_{k}^{2 m}(z, \bar{z})\right\}, \sqrt{2} \Im\left\{P_{k}^{2 m}(z, \bar{z})\right\}, 0 \leq k \leq\lfloor m\rfloor\right\}
\end{aligned}
$$

are real orthonormal polynomials and form a basis for $\mathcal{V}_{n}$ with $n=2 m-1$ or $2 m$, respectively.

5.2. Zeros of Chebyshev polynomials and Gaussian cubature formula. Let $w$ be a nonnegative weight function defined on a compact set $\Omega$ in $\mathbb{R}^{2}$. A cubature formula of degree $2 n-1$ is a sum of point evaluations that satisfies

$$
\int_{\Omega} f(x, y) w(x, y) d x d y=\sum_{j=1}^{N} \lambda_{j} f\left(x_{j}, y_{j}\right), \quad \lambda_{j} \in \mathbb{R}
$$

for every $f \in \Pi_{2 n-1}^{2}$, where $\Pi_{n}^{2}$ denote the space of polynomials of degree at most $n$ in two variables. The points $\left(x_{j}, y_{j}\right)$ are called nodes of the cubature and the numbers $\lambda_{i}$ are called weights. It is well-known that a cubature formula of degree $2 n-1$ exists only if $N \geq \operatorname{dim} \Pi_{n-1}^{2}$. A cubature that attains such a lower bound is called a Gaussian cubature.

Let $\mathbb{P}_{n}:=\left\{P_{k}^{n}: 0 \leq k \leq n\right\}$ denote a basis of orthonormal polynomials of degree $n$ with respect to $w(x, y) d x d y$. It is known that a Gaussian cubature exists if and only if $\mathbb{P}_{n}$ (every element in $\mathbb{P}_{n}$ ) has $\operatorname{dim} \Pi_{n-1}^{2}$ many common zeros, which are necessarily real and distinct. Furthermore, the weight $\lambda_{j}$ in a Gaussian cubature is given by

$$
\lambda_{j}=\left[K_{n-1}\left(x_{j}, y_{j}\right)\right]^{-1}, \quad K_{n}(x, y):=\sum_{k=0}^{n} \sum_{j=0}^{k} P_{j}^{k}(x) P_{j}^{k}(y) .
$$

The definition of $K_{n}(x, y)$ shows that it is the reproducing kernel of $\Pi_{n}^{2}$ in $L^{2}(\mu)$, so that it is independent of the choice of the basis.

Unlike the case of one variable, however, Gaussian cubatures do not exist in general. We refer to [4, 14, 20, for results and further discussions. It is sufficient to say that Gaussian cubatures are rare. In fact, the family of weight functions identified in [18, for which the Gaussian cubature exist for all $n$ remains the only known example up to now.

We now test the above theory on the generalized Chebyshev polynomials. Because of the relation (5.9), we can work with the zeros of the complex polynomials 
in $\mathbb{P}_{n}$. Furthermore, it follows from (5.4) that the reproducing kernel

$$
K_{n}(z, w):=\sum_{k=0}^{n} \mathbb{P}_{k}^{\operatorname{tr}}(z, \bar{z}) \mathbb{P}_{k}(w, \bar{w})=\sum_{k=0}^{n} \sum_{j=0}^{k} P_{j}^{k}(z, \bar{z}) P_{j}^{k}(w, \bar{w})
$$

is real and, by (5.9), is indeed the reproducing kernel of $\Pi_{n}^{2}$.

For the Chebyshev polynomials of the first kind, the polynomials in $\mathbb{P}_{n}$ do not have common zeros in general. For example, in the case of $m=2$, we have $T_{0}^{2}(z, \bar{z})=3 z^{2}-2 \bar{z}$ and $T_{1}^{2}(z, \bar{z})=(3 z \bar{z}-1) / 2$, so that the three real orthogonal polynomials of degree 2 can be given by

$$
\left\{3\left(x^{2}-y^{2}\right)-2 x, \quad 6 x y+2 y, \quad 3\left(x^{2}+y^{2}\right)-1\right\},
$$

which has no common zero at all. Therefore, there is no Gaussian cubature for the weigh function $w_{-\frac{1}{2}}$ on $\Delta^{*}$.

The Chebyshev polynomials of the second kind, on the other hand, does have the maximum number of common zeros, so that a Gaussian cubature exists for $w_{\frac{1}{2}}(\mathbf{t})$. Let

$$
X_{n}:=\Lambda_{n+2}^{\circ}=\left\{\left(\frac{k_{1}}{n+2}, \frac{k_{2}}{n+2}\right) \mid k_{1} \geq 1, k_{2} \geq 1, k_{1}+k_{2} \leq n+1\right\},
$$

which is in the interior of $\Delta$, and let $X_{n}^{*}$ denote the image of $X_{n}$ in $\Delta^{*}$ under the mapping (5.2).

Theorem 5.3. For the weight function $w_{\frac{1}{2}}$ on $\Delta^{*}$ a Gaussian cubature formula exists; that is,

$$
c_{\frac{1}{2}} \int_{\Delta^{*}} f(x, y) w_{\frac{1}{2}}(x, y) d x d y=\sum_{j_{1}=1}^{n+1} \sum_{j_{2}=1}^{n+1-j_{1}} \mu_{j}^{(n)} f\left(\frac{j_{1}}{n+2}, \frac{j_{2}}{n+2}\right), \quad \forall f \in \Pi_{2 n-1},
$$

where $j=\left(j_{1}, j_{2}\right)$,

$$
\mu_{j}^{(n)}=\frac{32}{9(n+2)^{2}} \sin ^{2} \pi \frac{j_{1}}{n+2} \sin ^{2} \pi \frac{j_{2}}{n+2} \sin ^{2} \pi \frac{j_{1}+j_{2}}{n+2} .
$$

Proof. The numerator of $U_{k}^{n}(\mathbf{t})$ is $\mathrm{TS}_{k+1, n-k+1,-n-2}(\mathbf{t})$, which is zero at all $\frac{j}{n+2}$ according to (4.3). The first equation of (3.15) shows that the denominator of $U_{k}^{n}(\mathbf{t})$ is

$$
\mathrm{TS}_{1,1,-2}(\mathbf{t})=\frac{4}{3} \sin \pi t_{1} \sin \pi t_{2} \sin \pi t_{3},
$$

which vanishes at the integer points on the boundary point of $\Delta$. Consequently, $U_{k}^{n}(\mathbf{t})$ does not vanish on the boundary of $\Delta$ but vanishes on $X_{n}$. It is easy to see that the cardinality of $X_{n}$ is $\operatorname{dim} \Pi_{n-1}^{2}$. Consequently, the corresponding $\mathbb{P}_{n}$ has $\operatorname{dim} \Pi_{n-1}^{2}$ common zeros in $X_{n}^{*}$, so that the Gaussian cubature with respect to $w_{\frac{1}{2}}$ exists, which takes the form (5.11). To obtain the formula for $\mu_{j}^{(n)}$, we note that the change of variables (5.2) shows that (5.11) is the same as

$$
\frac{1}{|\Delta|} \int_{\Delta} f(\mathbf{t})\left[\mathrm{TS}_{1,1,-2}(\mathbf{t})\right]^{2} d \mathbf{t}=\frac{2}{(n+2)^{2}} \sum_{j \in \Lambda_{n+2}^{\circ}}\left[\operatorname{TS}_{1,1,-2}\left(\frac{\mathbf{j}}{n+2}\right)\right]^{2} f\left(\frac{\mathbf{j}}{n+2}\right),
$$

where the last step follows from the fact that $\mathrm{TS}_{1,1,-2}(\mathbf{t})$ vanishes on the boundary of $\Delta$. This is exactly the cubature (4.13) applied to the function $f(\mathbf{t})\left[\operatorname{TS}_{1,1,-2}(\mathbf{t})\right]^{2}$. 
We note that $w_{\frac{1}{2}}$ is only the second example of a weight function for which a Gaussian cubature exists for all $n$. Furthermore, the example of $w_{-\frac{1}{2}}$ shows that the existence of Gaussian cubature does not hold for all weight functions in the family of $w_{\alpha}$. In [18], a family of weight functions depending on three parameters that are on a region bounded by a parabola and two lines is shown to ensure Gaussian cubature for all permissible parameters.

The set of nodes of a Gaussian cubature is poised for polynomial interpolation, which shows that there is a unique polynomial $P$ in $\Pi_{n-1}^{2}$ such that $P(\xi)=f(\xi)$ for all $\xi \in X_{n}^{*}$, where $f$ is an arbitrary function on $X_{n}^{*}$. In fact the general theory (cf. [4) immediately gives the following result:

Proposition 5.4. The unique interpolation polynomial of degree $n$ on $X_{n}^{*}$ is given by

$$
\mathcal{L}_{n} f(x)=\sum_{j_{1}=1}^{n+1} \sum_{j_{2}=1}^{n+1-j_{1}} f\left(\frac{j}{n}\right) \mu_{j}^{(n)} K_{n}\left(\mathbf{t}, \frac{j}{n}\right),
$$

where $x=\left(x_{1}, x_{2}\right)$ and $z(\mathbf{t})=x_{1}+i x_{2}$, and $\mathcal{L}_{n} f$ is of total degree $n-1$ in $x$.

Under the mapping (5.2), the above interpolation corresponds to a trigonometric interpolation on $\Lambda_{n+2}^{\circ}$. Since $\mu_{j}^{(n)}=\frac{2}{(n+2)^{2}}\left|\mathrm{TS}_{1,1,-2}\left(\frac{\mathbf{j}}{n}\right)\right|^{2}$, it follows readily that

$$
\mu_{j}^{(n)} K_{n}\left(\mathbf{t}, \frac{j}{n}\right)=\frac{2}{(n+2)^{2}} \mathrm{TS}_{1,1,-2}\left(\frac{\mathbf{j}}{n}\right) \sum_{\mathbf{k} \in \Lambda_{n+2}^{\circ}} \frac{\operatorname{TS}_{\mathbf{k}}(\mathbf{t}) \mathrm{TS}_{\mathbf{k}}\left(\frac{\mathbf{j}}{n}\right)}{\operatorname{TS}_{1,1,-2}(\mathbf{t})}
$$

where $\mathbf{j}=\left(j_{1}, j_{2},-j_{1}-j_{2}\right)$. Consequently, the interpolation (5.13) is closely related to the trigonometric interpolation on the triangle given in Theorem 4.6

5.3. Cubature formula and Chebyshev polynomials of the first kind. As discussed in the previous subsection, Gaussian cubature does not exist for $w_{-\frac{1}{2}}$ on $\Delta^{*}$. It turns out, however, that there is a Gauss-Lobatto type cubature for this weight function whose nodes are the points in the set

$$
Y_{n}:=\Lambda_{n}=\left\{\left(\frac{k_{1}}{n}, \frac{k_{2}}{n}\right) \mid k_{1} \geq 0, k_{2} \geq 0, k_{1}+k_{2} \leq n\right\},
$$

which is located inside $\Delta$. Again we let $Y_{n}^{*}$ denote the image of $Y_{n}$ in $\Delta^{*}$ under the mapping (5.2).

The cardinality of $Y_{n}$ is $\operatorname{dim} \Pi_{n}^{2}$. According to a characterization of cubature formula in the language of ideals and varieties (25]), there will be a cubature formula of degree $2 n-1$ based on $Y_{n}^{*}$ if there are $n+2$ linearly independent polynomials of degree $n+1$ that vanish on $Y_{n}^{*}$. In other words, $Y_{n}^{*}$ is the variety of an ideal generated by $n+2$ algebraic polynomials of degree $n+1$, which are necessarily quasi-orthogonal in the sense that they are orthogonal to all polynomials of degree $n-2$. Such an ideal and a basis are described in the following proposition.

Proposition 5.5. The set $Y_{n}^{*}$ is the variety of the polynomial ideal

$$
\left\langle T_{0}^{n+1}-T_{1}^{n}, \quad T_{k}^{n+1}-T_{k-1}^{n-1} \quad(1 \leq k \leq n), \quad T_{n+1}^{n+1}-T_{n-1}^{n}\right\rangle .
$$


Proof. The mapping (5.2) allows us to work with $T_{k}^{n}(\mathbf{t})$ on $Y_{n}$. Using the explicit formula for $\mathrm{TC}_{k, n-k,-n}$ in (4.2), we have

$$
\begin{aligned}
T_{k}^{n}(\mathbf{t})= & \frac{1}{3}\left[e^{\frac{i \pi}{3}(n-2 k)\left(t_{2}-t_{3}\right)} \cos n \pi t_{1}\right. \\
& \left.+e^{\frac{i \pi}{3}(n-2 k)\left(t_{3}-t_{1}\right)} \cos n \pi t_{2}+e^{\frac{i \pi}{3}(n-2 k)\left(t_{1}-t_{2}\right)} \cos n \pi t_{3}\right] .
\end{aligned}
$$

Since $n+1-2 k=n-1-2(k-1)$, the above formula implies that

$$
\begin{aligned}
T_{k}^{n+1}(\mathbf{t})-T_{k-1}^{n-1}(\mathbf{t})=\frac{-1}{3} & {\left[e^{\frac{i \pi}{3}(n-2 k)\left(t_{2}-t_{3}\right)} \sin \pi t_{1} \sin m \pi t_{1}\right.} \\
& +e^{\frac{i \pi}{3}(n-2 k)\left(t_{3}-t_{1}\right)} \sin \pi t_{2} \sin n \pi t_{2} \\
& \left.+e^{\frac{i \pi}{3}(n-2 k)\left(t_{1}-t_{2}\right)} \sin \pi t_{3} \sin n \pi t_{3}\right],
\end{aligned}
$$

from which it follows immediately that $T_{k}^{n+1}-T_{k-1}^{n+1}$ vanishes on $Y_{n}$ for $1 \leq k \leq n$. Furthermore, a tedious computation shows that

$$
\begin{aligned}
& T_{k}^{n}\left(\frac{j_{1}}{n}, \frac{j_{2}}{n}, \frac{-j_{1}-j_{2}}{n}\right)=\frac{-1}{6} e^{\frac{-2 i \pi}{3 n}(n+1) j_{1}+j_{2}}\left[\left(-2+e^{2 i \pi j_{2}}+e^{2 i \pi\left(j_{1}+j_{2}\right)}\right)\right. \\
& \left.+e^{2 i \pi \frac{j_{2}}{n}}\left(-2 e^{2 i \pi j_{2}}+e^{2 i \pi\left(j_{1}+j_{2}\right)}+1\right)+e^{2 i \pi \frac{j_{1}+j_{2}}{n}}\left(-2 e^{2 i \pi j_{1}}+e^{2 i \pi j_{2}}+1\right)\right],
\end{aligned}
$$

which is evidently zero whenever $j_{1}$ and $j_{2}$ are integers. Finally, we note that $T_{n+1}^{n+1}-T_{n-1}^{n}$ is the conjugate of $T_{0}^{n+1}-T_{1}^{n}$. This completes the proof.

As mentioned before, this proposition already implies that a cubature formula of degree $2 n-1$ based on the nodes of $Y_{n}^{*}$ exists.

Theorem 5.6. For the weight function $w_{-\frac{1}{2}}$ on $\Delta^{*}$ the cubature formula

$$
c_{-\frac{1}{2}} \int_{\Delta^{*}} f(x, y) w_{-\frac{1}{2}}(x, y) d x d y=\frac{1}{3 n^{2}} \sum_{k_{1}=0}^{n} \sum_{k_{2}=0}^{k_{1}} \lambda_{k}^{(n)} f\left(\frac{k_{1}}{n}, \frac{k_{2}}{n}\right), \quad \forall f \in \Pi_{2 n-1},
$$

holds, where $\lambda_{k}^{(n)}=\lambda_{k, n-k,-n}^{(n)}$ with $\lambda_{\mathbf{j}}^{(n)}$ given by (4.9).

In fact, the change of variables back to $\Delta$ shows that the cubature (5.15) is exactly of the form (4.13), which gives the values of $\lambda_{k}^{(n)}$. In particular, this gives a proof of Theorem 4.5 .

Another way of determining the cubature weight $\lambda_{k}^{(n)}$ is to work with the reproducing kernel and the polynomial ideal. This approach also gives another way of determining the formula for polynomial interpolation. Furthermore, it fits into a general framework (see 24] and the references therein), and the intermediate results are of independent interest. In the rest of this section, we follow through with this approach.

The recursive relation (5.5) for $T_{k}^{m}$ can be conveniently written in matrix form. Recall that for $T_{k}^{m}$, the orthonormal polynomials are

$$
P_{0}^{m}=\sqrt{3} T_{0}^{m}, \quad P_{k}^{m}=\sqrt{6} T_{k}^{m} \quad(1 \leq k \leq m-1), \quad P_{m}^{m}=\sqrt{3} T_{m}^{m}
$$


for $m>0$ and $P_{0}^{0}=1$. In the following we treat $\mathbb{P}_{m}$ defined in (5.8) as a column vector. The relation (5.4) shows an important relation

$$
\overline{\mathbb{P}_{m}}=J_{m+1} \mathbb{P}_{m}, \quad J_{m}=\left[\begin{array}{lll}
\bigcirc & & 1 \\
& . & \\
1 & & \bigcirc
\end{array}\right] .
$$

Using these notations, we have the following three-term relations:

Proposition 5.7. Define $\mathbb{P}_{-1}=0$. For $m \geq 0$,

$$
\begin{aligned}
& z \mathbb{P}_{m}=A_{m} \mathbb{P}_{m+1}+B_{m} \mathbb{P}_{m}+C_{m} \mathbb{P}_{m-1}, \\
& \bar{z} \mathbb{P}_{m}=C_{m+1}^{\mathrm{tr}} \mathbb{P}_{m+1}+B_{m}^{\mathrm{tr}} \mathbb{P}_{m}+A_{m-1}^{\mathrm{tr}} \mathbb{P}_{m-1},
\end{aligned}
$$

where $A_{m}, B_{m}$ and $C_{m}$ are given by

$$
A_{m}=\frac{1}{3}\left[\begin{array}{ccccc}
1 & & & \bigcirc & 0 \\
& \ddots & & & \vdots \\
& & 1 & & \\
& & & \sqrt{2} & 0
\end{array}\right], B_{m}=\frac{1}{3}\left[\begin{array}{cccccc}
0 & \sqrt{2} & 0 & \ldots & & \bigcirc \\
0 & & 1 & & \\
\vdots & & & \ddots & & \\
0 & \bigcirc & & & 1 & 0 \\
0 & 0 & \ldots & & 0 & \sqrt{2}
\end{array}\right] .
$$

and $C_{m}=J_{m} A_{m-1}^{\text {tr }} J_{m-1}$.

In fact, the first relation is exactly (5.5). To verify the second relation, take the complex conjugate of the first equation and then use (5.16).

In the following we slightly abuse the notation by writing $\mathbb{P}_{n}(\mathbf{t})$ in place of $\mathbb{P}_{n}(z)$, when $z=z(\mathbf{t})$ is given by (5.1). Similarly, we write $K_{n}(\mathbf{t}, \mathbf{s})$ in place of $K_{n}(z, w)$ when $z=z(\mathbf{t})$ and $w=w(\mathbf{s})$. Just like the case of real orthogonal polynomials, the three-term relation implies a Christoph-Darboux type formula (4])

Proposition 5.8. For $m \geq 0$, we have

$$
(z-w) K_{n}(z, w):=\mathbb{P}_{n+1}^{\mathrm{tr}}(z) A_{n}^{\mathrm{tr}} \overline{\mathbb{P}_{n}(w)}-\mathbb{P}_{n}^{\mathrm{tr}}(z) C_{n+1}^{\mathrm{tr}} \overline{\mathbb{P}_{n+1}(w)} .
$$

Next we incorporate the information on the ideal in (5.14) into the kernel function. The $n+2$ polynomials in the ideal can be written as a set

$$
\mathbb{Q}_{n+1}=\mathbb{P}_{n+1}-\Gamma_{0} \mathbb{P}_{n}-\Gamma_{1} \mathbb{P}_{n-1},
$$

which we also treat as a column vector below, where

$$
\Gamma_{0}=\left[\begin{array}{ccccc}
0 & \frac{1}{\sqrt{2}} & 0 & \ldots & 0 \\
0 & \cdots & \bigcirc & \cdots & \bigcirc \\
0 & \cdots & 0 & \frac{1}{\sqrt{2}} & 0
\end{array}\right] \text { and } \Gamma_{1}=\left[\begin{array}{ccccc}
0 & \ldots & 0 & \ldots & 0 \\
\sqrt{2} & & & & \bigcirc \\
& 1 & & & \\
& & \ddots & & \\
& & & 1 & \\
0 & & & & \sqrt{2} \\
0 & \ldots & 0 & \ldots & 0
\end{array}\right]
$$

Proposition 5.9. For $n \geq 0$, define

$$
\Phi_{n}(\mathbf{t}, \mathbf{s}):=\frac{1}{2}\left[K_{n}(\mathbf{t}, \mathbf{s})+K_{n-1}(\mathbf{t}, \mathbf{s})\right]-\frac{1}{2}\left[T_{0}^{n}(\mathbf{t}) \overline{T_{0}^{n}(\mathbf{s})}+\overline{T_{0}^{n}(\mathbf{t})} T_{0}^{n}(\mathbf{s})\right],
$$

and we again write $\Phi_{n}(z, w)$ in place of $\Phi_{n}(\mathbf{t}, \mathbf{s})$ for $z=z(\mathbf{t})$ and $w=w(\mathbf{s})$. Then

$$
(z-w) \Phi_{n}(z, w)=\mathbb{Q}_{n+1}^{\mathrm{tr}}(z) A_{n}^{\mathrm{tr}} M_{n} \overline{\mathbb{P}_{n}(w)}-\mathbb{P}_{n}^{\mathrm{tr}}(z) M_{n} C_{n+1}^{\mathrm{tr}} \overline{\mathbb{Q}_{n+1}(w)}
$$

where $M_{n}=\operatorname{diag}\{-1 / 3,1 / 2, \ldots, 1 / 2,-1 / 3\}$ is a diagonal matrix. 
Proof. Inserting $\mathbb{P}_{n+1}=\mathbb{Q}_{n+1}+\Gamma_{0} \mathbb{P}_{n}+\Gamma_{1} \mathbb{P}_{n-1}$ into (5.18) gives

$$
\begin{aligned}
(z-w) K_{n}(z, w)= & \mathbb{Q}_{n+1}^{\operatorname{tr}}(z) A_{n}^{\operatorname{tr}} \overline{\mathbb{P}_{n}(w)}-\mathbb{P}_{n}^{\operatorname{tr}}(z) C_{n+1}^{\operatorname{tr}} \overline{\mathbb{Q}_{n+1}(w)} \\
& +\mathbb{P}_{n}^{\operatorname{tr}}(z) \Gamma_{0}^{\operatorname{tr}} A_{n}^{\operatorname{tr}} \overline{\mathbb{P}_{n}(w)}-\mathbb{P}_{n}^{\operatorname{tr}}(z) C_{n+1}^{\operatorname{tr}} \Gamma_{0} \overline{\mathbb{P}_{n}(w)} \\
& +\mathbb{P}_{n-1}^{\operatorname{tr}}(z) \Gamma_{1}^{\operatorname{tr}} A_{n}^{\operatorname{tr}} \overline{\mathbb{P}_{n}(w)}-\mathbb{P}_{n}^{\operatorname{tr}}(z) C_{n+1}^{\operatorname{tr}} \Gamma_{1} \overline{\mathbb{P}_{n-1}(w)}
\end{aligned}
$$

Let $D_{n}$ be a $n \times n$ matrix. Then similarly we have

$$
\begin{aligned}
& (z-w) \mathbb{P}_{n}^{\operatorname{tr}}(z) D_{n} \overline{\mathbb{P}_{n}(w)}=\mathbb{Q}_{n+1}^{\operatorname{tr}}(z) A_{n}^{\operatorname{tr}} D_{n} \overline{\mathbb{P}_{n}(w)}-\mathbb{P}_{n}^{\operatorname{tr}}(z) D_{n} C_{n+1}^{\operatorname{tr}} \overline{\mathbb{Q}_{n+1}(w)} \\
& \quad+\mathbb{P}_{n}^{\operatorname{tr}}(z)\left(A_{n} \Gamma_{0}+B_{n}\right)^{\operatorname{tr}} D_{n} \overline{\mathbb{P}_{n}(w)}-\mathbb{P}_{n}^{\operatorname{tr}}(z) D_{n}\left(C_{n+1}^{\operatorname{tr}} \Gamma_{0}+B_{n}^{\operatorname{tr}}\right) \overline{\mathbb{P}_{n}(w)} \\
& \quad+\mathbb{P}_{n-1}^{\operatorname{tr}}(z)\left(A_{n} \Gamma_{1}+C_{n}\right)^{\operatorname{tr}} D_{n} \overline{\mathbb{P}_{n}(w)}-\mathbb{P}_{n}^{\operatorname{tr}}(z) D_{n}\left(C_{n+1}^{\mathrm{tr}} \Gamma_{1}+A_{n-1}^{\operatorname{tr}}\right) \overline{\mathbb{P}_{n-1}(w)}
\end{aligned}
$$

In order to prove the stated result, we take the difference of the two equations and choose $D_{n}$ such that

$$
\begin{aligned}
& \left(A_{n} \Gamma_{0}+B_{n}\right)^{\mathrm{tr}} D_{n}-D_{n}\left(C_{n+1}^{\mathrm{tr}} \Gamma_{0}+B_{n}^{\mathrm{tr}}\right)=\Gamma_{0}^{\mathrm{tr}} A_{n}^{\mathrm{tr}}-C_{n+1}^{\mathrm{tr}} \Gamma_{1}, \\
& \left(A_{n} \Gamma_{1}+C_{n}\right)^{\mathrm{tr}} D_{n}=\Gamma_{1}^{\mathrm{tr}} A_{n}^{\mathrm{tr}}, \quad D_{n}\left(C_{n+1}^{\mathrm{tr}} \Gamma_{1}+A_{n-1}^{\mathrm{tr}}\right)=C_{n+1}^{\mathrm{tr}} \Gamma_{1} .
\end{aligned}
$$

It turns out that the unique solution of the above three equations is given by $D_{n}=\operatorname{diag}\{2 / 3,1 / 2,1 / 2, \ldots, 1 / 2,2 / 3\}$, which is completely determined by the last two equations and then verified to satisfy the first equation. As a consequence of this choice of $D_{n}$, we obtain

$$
\begin{aligned}
(z-w)\left[K_{n}(z, w)-\mathbb{P}_{n}^{\mathrm{tr}}(z) D_{n} \overline{\mathbb{P}(w)}\right] & =\mathbb{Q}_{n+1}^{\mathrm{tr}}(z) A_{n}^{\mathrm{tr}}\left(I-D_{n}\right) \overline{\mathbb{P}_{n}(w)} \\
& -\mathbb{P}_{n}^{\mathrm{tr}}(z)\left(I-D_{n}\right) C_{n+1}^{\mathrm{tr}} \overline{\mathbb{Q}_{n+1}(w)}
\end{aligned}
$$

where $I$ denotes the identity matrix. Setting $M_{n}=I-D_{n}$ we see that the right hand side agrees with the right hand side of (5.20). Finally we note that

$$
\begin{aligned}
\mathbb{P}_{n}^{\operatorname{tr}}(z) D_{n} & \overline{\mathbb{P}_{n}(w)}=\frac{1}{2} \mathbb{P}_{n}^{\operatorname{tr}}(z) \overline{\mathbb{P}_{n}(w)}+\left[P_{0}^{n}(z) \overline{P_{0}^{n}(w)}+P_{n}^{n}(z) \overline{P_{n}^{n}(w)}\right] \\
= & \frac{1}{2}\left[K_{n}(z, w)-K_{n-1}(z, w)\right]+\frac{1}{2}\left[T_{0}^{n}(z) \overline{T_{0}^{n}(w)}+\overline{T_{0}^{n}(z)} T_{0}^{n}(z)\right],
\end{aligned}
$$

which completes the proof.

As a consequence of the above proposition, it is readily seen that $\Phi_{n}\left(\frac{j}{n}, \frac{k}{n}\right)=0$ for $j \neq k$. Furthermore, we note that $\Phi_{n}(\mathbf{t}, \mathbf{s})$ is in fact a real valued function. Consequently, we have proved the following.

Proposition 5.10. The unique interpolation polynomial on $Y_{n}^{*}$ is given by

$$
\mathcal{L}_{n}^{*} f(x, y)=\sum_{j_{1}=0}^{n} \sum_{j_{2}=0}^{n-j_{1}} f\left(\frac{j}{n}\right) \frac{\Phi_{n}\left(\mathbf{t}, \frac{j}{n}\right)}{\Phi_{n}\left(\frac{j}{n}, \frac{j}{n}\right)}
$$

where $z(\mathbf{t})=x+i y$, and $\mathcal{L}_{n}^{*} f$ is of total degree $n$ in $x$ and $y$.

In fact, upon changing variables (5.1), this interpolation polynomial is exactly the trigonometric interpolation function $\mathcal{L}_{n}^{*} f(\mathbf{t})$ at (4.14). Here the uniqueness of the interpolation follows from the general theory. Moreover, integrating $\mathcal{L}_{n}^{*} f$ with respect to $w_{-\frac{1}{2}}$ over $\Delta^{*}$ yields the cubature formula (5.15) with

$$
\lambda_{j}^{(n)}=\left[\Phi_{n}\left(\frac{j}{n}, \frac{j}{n}\right)\right]^{-1},
$$


which can be used to determine $\lambda_{j}^{(n)}$ if needed. The function $K_{n}(\mathbf{t}, \mathbf{s})$, thus $\Phi_{n}(\mathbf{t}, \mathbf{s})$, enjoys a compact formula, which facilitates the computation of $\Phi_{n}\left(\frac{j}{n}, \frac{j}{n}\right)$. The compact formula follows from the following identity.

Proposition 5.11. Let $D_{n}$ be defined in (3.12). Then

$$
K_{n}(\mathbf{s}, \mathbf{t})=\sum_{k=0}^{n} \sum_{j=0}^{k} \widetilde{T}_{j}^{k}(\mathbf{t}) \overline{\widetilde{T}_{j}^{k}(\mathbf{s})}=\mathcal{P}_{t}^{+} D_{n}(\mathbf{t}-\mathbf{s}) .
$$

The identity can be established from the definition of $T_{j}^{k}$ and $D_{n}$ as in the proof of Theorem 4.6 upon using the fact that $\mathcal{P}_{\mathbf{t}}^{+} \mathcal{P}_{\mathbf{s}}^{+} F(\mathbf{t}-\mathbf{s})=\mathcal{P}^{+} F(\mathbf{t}-\mathbf{s})$. We omit the details. From this identity and (5.22), we immediately have a compact formula for the interpolation polynomial, whose trigonometric counterpart under the change of variables (5.2) is exactly the function $\ell_{\mathbf{j}, n}^{\Delta}$ in Theorem 4.7

Acknowledgment. The authors thank the referees for their meticulous and prudent comments.

\section{REFERENCES}

[1] V. V. Arestov and E. E. Berdysheva, Turán's problem for positive definite functions with supports in a hexagon, Proc. Steklov Inst. Math. 2001, Approximation Theory. Asymptotical Expansions, suppl. 1, S20-S29.

[2] J. H. Conway and N. J. A. Sloane, Sphere Packings, Lattices and Groups, 3rd ed. Springer, New York, 1999.

[3] D. E. Dudgeon and R. M. Mersereau, Multidimensional Digital Signal Processing, PrenticeHall Inc, Englewood Cliffs, New Jersey, 1984.

[4] C. F. Dunkl and Yuan Xu, Orthogonal polynomials of several variables, Encyclopedia of Mathematics and its Applications, vol. 81, Cambridge Univ. Press, 2001.

[5] W. Ebeling, Lattices and Codes, Vieweg, Braunschweig/Wiesbaden, 1994.

[6] B. Fuglede, Commuting self-adjoint partial differential operators and a group theoretic problem, J. Functional Anal. 16 (1974), 101-121.

[7] J. R. Higgins, Sampling theory in Fourier and Signal Analysis, Foundations, Oxford Science Publications, New York, 1996.

[8] M. Kolountzakis, The study of translation tiling with Fourier analysis, Fourier analysis and convexity, 131-187, Appl. Numer. Harmon. Anal., Birkhuser Boston, Boston, MA, 2004.

[9] T. Koornwinder, Orthogonal polynomials in two varaibles which are eigenfunctions of two algebraically independent partial differential operators, Nederl. Acad. Wetensch. Proc. Ser. A77 = Indag. Math. 36 (1974), 357-381.

[10] T. Koornwinder, Two-variable analogues of the classical orthogonal polynomials, in Theory and applications of special functions, 435-495, ed. R. A. Askey, Academic Press, New York, 1975.

[11] R. J. Marks II, Introduction to Shannon Sampling and Interpolation Theory, Springer-Verlag, New York, 1991.

[12] B. J. McCartin, Eigenstructure of the equilateral triangle, Part I: The Dirichlet problem, SIAM Review, 45 (2003), 267-287.

[13] B. J. McCartin, Eigenstructure of the equilateral triangle, Part II: The Neumann problem, Math. Prob. Engineering, 8 (2002), 517-539.

[14] I. P. Mysoviskikh, Interpolatory cubature formulas, Nauka, Moscow, 1981.

[15] M. A. Pinsky, The eigenvalues of an equilateral triangle, SIAM J. Math. Anal., 11 (1980), 819-827.

[16] M. A. Pinsky, Completeness of the eigenfunctions of the equilateral triangle, SIAM J. Math. Anal., 16 (1985), 848-851.

[17] T. J. Rivlin, An introduction to the approximation of functions, Dover Publ., New York, 1981.

[18] H. J. Schmid and Yuan Xu, On bivariable Gaussian cubature formulae, Proc. Amer. Math. Soc. 122 (1994), 833-842. 
[19] I. H. Sloan and T. R. Osborn, Multiple integration over bounded and unbounded regions, J. Comp. Applied Math. 17 (1987) 181-196.

[20] A. Stroud, Approximate calculation of multiple integrals, Prentice-Hall, Englewood Cliffs, NJ, 1971.

[21] J. Sun, Multivariate Fourier series over a class of non tensor-product partition domains, $J$. Comput. Math. 21 (2003), 53-62.

[22] J. Sun and H. Li, Generalized Fourier transform on an arbitrary triangular domain, Adv. Comp. Math., 22 (2005), 223-248.

[23] G. Szegö, Orthogonal Polynomials, Amer. Math. Soc. Colloq. Publ. Vol.23, Providence, 4th edition, 1975.

[24] Yuan Xu, On orthogonal polynomials in several variables, Special Functions, q-series and Related Topics, The Fields Institute for Research in Mathematical Sciences, Communications Series, Volume 14, 1997, p. 247-270.

[25] Yuan $\mathrm{Xu}$, Polynomial interpolation in several variables, cubature formulae, and ideals, $A d$ vances in Comp. Math., 12 (2000), 363-376.

[26] A. Zygmund, Trigonometric series, Cambridge Univ. Press, Cambridge, 1959.

Institute of Software, Chinese Academy of Sciences, Beijing 100080, China

E-mail address: hynli@mail.rdcps.ac.cn

Institute of Software, Chinese Academy of Sciences, Beijing 100080,China

E-mail address: sun@mail.rdcps.ac.cn

Department of Mathematics, University of Oregon, Eugene, Oregon 97403-1222.

E-mail address: yuan@math.uoregon.edu 